\title{
GRADO DE PENETRACIÓN Y DIFUSIÓN DE TRES ANGLICISMOS EN EL ESPAÑOL DE MÉXICO*
}

\author{
Erik Daniel Franco Trujillo \\ El Colegio de México \\ efranco@colmex.mx
}

This paper describes the degree of penetration and diffusion of the following lexical anglicisms in Mexican Spanish: bullying, gay, and checar. We examine both linguistic and social indicators to find out in which way a lexical item is introduced into a speech community and expands throughout different social and linguistic contexts. We search for occurrences of these lexical items in different linguistic corpora, as well as in the search engine Google. The findings are further supported with a survey questionnaire that considers social variables such as gender, age, and education level. With this methodology, we try to combine production and competence data in order to describe the degree of penetration and diffusion of these loanwords in Mexican Spanish.

Keywords: lexical anglicism, diffusion, Mexican Spanish

* Quiero agradecer a Pedro Martín Butragueño por su ayuda durante la realización de este trabajo. Sin su apoyo constante e incondicional hubiera sido imposible llevar a cabo esta investigación. 
En este trabajo se describe el grado de penetración y difusión de los anglicismos bullying, gay y checar en el español de México, a través de una serie de indicadores tanto lingüísticos como sociales. Se muestran los resultados obtenidos de una serie de búsquedas de estas unidades léxicas en diferentes corpus lingüísticos, así como en el buscador Google. Los hallazgos obtenidos se complementan con un cuestionario que considera las variables sociales de género, edad y escolaridad. A través de esta metodología se trata de describir el grado de penetración y difusión de estos préstamos léxicos en el español mexicano.

Palabras clave: anglicismo léxico, difusión, español de México

\section{INTRODUCCIÓN}

Sin lugar a dudas, la riqueza léxica del español refleja el contacto que sus hablantes han establecido a lo largo del tiempo con hablantes de otras lenguas y culturas. El impacto de estos encuentros es de tal importancia que, como bien señala Pedro Álvarez de Miranda (2004:1037), una de las tres dimensiones en la que se puede dividir el vocabulario de una lengua es la de los préstamos. ${ }^{1}$ Por préstamo debe entenderse "tout procédé par lequel les utilisateurs d'une langue adoptent intégralement ou partiellement une unité ou un trait linguistique (lexical, sémantique, morphologique, syntaxique, phonétique) d'une autre langue” (Office Québécois de la Langue Française 2007).

De la variedad de préstamos lingüísticos que se pueden presentar, el préstamo léxico es el más común de todos (Moreno de Alba 1992:196). El préstamo léxico, a diferencia de otros tipos de préstamos como los calcos y los préstamos semánticos, se caracteriza por ser una adopción integral,

1 Los otros dos sectores los constituyen las palabras patrimoniales y las palabras creadas por medio de procesos de derivación, composición y parasíntesis. 
es decir, lo que pasa de una lengua fuente a una lengua meta es un signo lingüístico en su totalidad. ${ }^{2}$ La importancia de este fenómeno ha llevado a algunos a afirmar que en el vocabulario de una lengua particular hay más préstamos que palabras patrimoniales (Sala 1988:159). Más allá de preguntarnos si dicha afirmación es válida en todos los casos, queda claro que la influencia extranjera es una de las causas principales del cambio léxico (Ullmann 1965). ${ }^{3}$

$\mathrm{Al}$ hacer un breve recorrido de la historia del español, encontramos la presencia de voces latinas y árabes en la Edad Media (Lapesa 1942:79), la incorporación de galicismos en el ámbito político, militar, cultural y tecnológico desde el medioevo hasta principios del siglo xx (Curell 2006) y la aparición de voces amerindias en Hispanoamérica como consecuencia de la conquista (Mejías 1980). Además, desde mediados del siglo xx hasta nuestros días, gracias a la expansión militar y económica de los Estados Unidos, la lengua que mayor influencia ha tenido sobre el español, en términos léxicos, es el inglés.

2 Se cuenta con un buen número de trabajos que proponen tipologías para acercarse al estudio de las diferentes características que los préstamos pueden tener. Para cuestiones generales sobre el préstamo vale la pena consultar propuestas como la de Haugen (1950) y Humbley (1974); por su parte, existen propuestas tipológicas como las de Pratt (1980), Gómez Capuz (1991) y Lorenzo (1996) que se centran exclusivamente en el anglicismo.

3 Si bien el interés de Ullmann se centra en las causas del cambio semántico, creemos que su clasificación resulta útil para comprender la dinámica del cambio léxico. En este sentido, Ullmann (1962), inspirado en las propuestas de Meillet (1926) y Sperber (1926), distingue seis grandes causas que están detrás de cualquier cambio de significado: causas lingüísticas, causas históricas, causas sociales, causas psicológicas, influencia extranjera y exigencia de un nuevo nombre. 
Tener en cuenta que el caudal léxico de una lengua se enriquece constantemente a través de la adopción de préstamos también es un recordatorio del dinamismo y movilidad que caracteriza al vocabulario de las lenguas (Otaola 2004:59-60). Por algo se ha reconocido que el plano léxico es el subsistema lingüístico que se transforma con mayor rapidez, pues "está en íntimo contacto con el mundo extralingüístico cuyos cambios políticos, económicos, sociales y culturales refleja más o menos directamente" (Schifko1992:143).

Para comprender el dinamismo constante del léxico de las lenguas, Schifko (1992) destaca tres procedimientos neológicos de los que se valen los hablantes para adaptarse a nuevas situaciones y realidades: los cambios semánticos, el préstamo y la formación de palabras. Estos procedimientos responden a tres principios básicos: satisfacer las necesidades comunicativas y expresivas de los hablantes, contar con las herramientas necesarias para nombrar los aspectos objetivos cambiantes del mundo y mantener la funcionalidad estructural de la lengua para alcanzar estos fines.

La complejidad que entraña la dinámica del cambio léxico resalta la necesidad de entender el fenómeno tanto desde una perspectiva lingüística, como desde una perspectiva social, en particular si tomamos en cuenta que el acervo léxico de las lenguas representa la memoria colectiva, la cosmovisión y la identidad cultural de sus hablantes. ${ }^{4}$

Si nos ceñimos exclusivamente al caso de los préstamos léxicos, el interés social y cultural que acarrean no hace sino volverse aún más evidente, pues todo intercambio lingüístico de esta índole involucra, por lo menos, el contacto de dos lenguas y dos culturas. Como es sabido, el contacto entre

\footnotetext{
4 Para más al respecto ver Lara (1990).
} 
lenguas se puede establecer de manera directa o de manera indirecta (Sala 1988:17). Un contacto directo es aquel que "se establece en el mismo territorio, a través de la mezcla de poblaciones o la convivencia durante un periodo variable", mientras que un contacto indirecto se manifiesta en territorios o regiones diferentes "a través de relaciones culturales, económicas y políticas". Evidentemente, las consecuencias lingüísticas de estos dos tipos de contactos difieren entre sí. El contacto directo suele implicar una etapa de bilingüismo en la que, en última instancia, se generan fenómenos de interferencia que pueden afectar los dominios más estructurados de una lengua, a saber, la fonología, la morfología, la sintaxis y algunas zonas del vocabulario (Mackey 1970). Por su parte, en el contacto indirecto, el tipo de intercambios lingüísticos que se da entre dos comunidades lingüísticas suele tener consecuencias exclusivamente en el plano léxico y sintáctico (Sala 1988).

Creemos que un contacto como el que existe entre el inglés y el español mexicano es sobre todo un contacto de tipo indirecto y, por lo mismo, se produce y expande a través de los medios masivos de comunicación como la radio, la televisión o Internet (Lázaro Carreter 1987). Tomando estas premisas como punto de partida, el propósito de este trabajo es analizar el momento de expansión en el que se encuentran algunos préstamos léxicos del inglés en el español de México, apelando a la idea de que cada palabra tiene un patrón evolutivo particular.

La investigación surge de la inquietud de buscar un método que permita trazar el grado de penetración y difusión de dichas palabras apelando tanto a indicadores lingüísticos como sociales. ${ }^{5}$ En consecuencia, se

\footnotetext{
5 Por difusión debe entenderse un cambio entre comunidades de habla (Labov 2010).
} 
considera el fenómeno del préstamo como un problema sociolingüístico en el que una pieza léxica es introducida por los hablantes para luego sufrir un proceso de expansión por medio de ciertos contextos lingüísticos, tanto orales como escritos, así como en ciertos ámbitos sociales. Desde el enfoque que se maneja en este estudio, el grado de penetración implica:

a) Observar qué tan acoplado está un préstamo al comportamiento morfológico del español.

b) Observar qué tan estable es el significado del préstamo estudiado entre los hablantes.

c) Observar el grado de expansión del uso del préstamo en distintos contextos lingüísticos y sociales.

A este respecto, se consideran tres niveles de búsqueda para fundamentar la reconstrucción del proceso de integración al español mexicano de los siguientes anglicismos léxicos: bullying, gay y checar.

\section{Metodología}

La elección de estas palabras se llevó a cabo con base en los siguientes criterios:

I. Evitar elegir palabras como DVD, walkman, Internet, iPod, entre otras, ya que resultaban poco atractivas para el estudio si se considera que la palabra se difunde tan pronto se difunde el objeto mismo. Dicho esto, resulta hasta cierto punto predecible dar cuenta del proceso de expansión del término, ya que se puede ir directamente al momento de aparición del objeto en cuestión para calcular el inicio del uso de la palabra. 
II. Partir de un nivel hipotético de antigüedad con la idea de formar un continuum en cuyos extremos se encontraran términos de largo recorrido por un lado y términos de reciente aparición por el otro. A este respecto, la palabra checar se colocó en el extremo de los términos que se cree tienen una larga trayectoria en el idioma; bullying se colocó en el extremo de los términos de reciente aparición y gay se colocó a la mitad de dicho continuum.

Una vez seleccionadas las unidades léxicas a estudiar, se realizó la búsqueda de datos en tres dimensiones distintas. En primer lugar, se buscaron las ocurrencias de cada una de estas piezas en los siguientes corpus: el Corpus Sociolingüistico de la Ciudad de México (CSCM), el Corpus Diacrónico del Español (CORDE), el Corpus de Referencia del Español Actual (CREA), el Corpus del Español Mexicano Contemporáneo (CEMC) y el Corpus del Español de Mark Davies (CEMD). ${ }^{6}$ La búsqueda en el CSCM permitió documentar la difusión social de las voces consideradas en un corpus oral, pues se trata de 108 entrevistas sociolingüísticas en las que se consideran las variables sociales de género, edad y grado de instrucción. ${ }^{7}$ Las búsquedas en los otros corpus permitieron documentar el proceso de expansión

${ }^{6}$ Evidentemente, para el CORDE, el CREA y el Corpus del Español de Mark Davies, se acotaron los criterios de búsqueda a contextos de uso exclusivamente de México.

7 A este respecto, el género comprende hombres y mujeres, la edad considera 3 generaciones: la primera formada por personas de entre 20 y 34 años de edad; la segunda conformada por personas de entre 35 y 54 ańos y la tercera incluye individuos de 55 años en adelante. Por último, el grado de instrucción se divide en bajo, medio y alto. El grado de instrucción bajo comprende personas que tienen como máximo educación básica. El grado de instrucción medio comprende personas que tienen como máximo la enseñanza media. El grado de instrucción alto considera individuos con educación superior. 
de las palabras en cuestión tanto en lengua oral como en lengua escrita. En el caso particular de estas búsquedas, se procuró anotar la fecha más antigua de aparición de los términos estudiados, así como algunos de los géneros en los que se documentó su ocurrencia.

En segundo lugar, dado que los resultados de esta exploración en distintos corpus no siempre resultaron lo suficientemente fructíferos, el siguiente paso fue continuar el proceso de recopilación de datos en el Diccionario del Español de México (DEM) y en el buscador Google. Dado que uno de los objetivos de la investigación es dar cuenta del proceso progresivo de expansión de estas palabras, se optó por investigar el crecimiento en cuanto al número de usuarios de Internet en México a lo largo de los años, así como ubicar el año en que Internet hizo su apertura comercial en el país para poder medir de alguna manera la proporción de la expansión del término. De acuerdo con Gutiérrez Cortés e Islas Carmona (2008), a partir de 1995 es cuando Internet comenzó su expansión comercial en la República Mexicana. Los criterios de búsqueda en Google se acotaron solamente a páginas de México; además, gracias a que el buscador Google permite realizar búsquedas fechadas, se tomaron en consideración tres lapsos de tiempo distintos dentro de los cuales se realizaron las búsquedas deseadas. El primero de estos lapsos va del 01/01/1995 al 31/12/2000; el segundo, del 01/01/2001 al 31/12/2006; por último, el tercer lapso de tiempo cubre el periodo del 01/01/2007 al 20/09/2012. Como se puede observar, se trata de un continuo de 15 ańos dividido en tres periodos de tiempo de cinco años cada uno. La razón principal para hacer estos cortes temporales fue contar con un contexto de fondo a partir del cual se pudiera medir la expansión relativa de las unidades léxicas de nuestro interés. A continua- 
ción, se hizo una búsqueda general del 01/01/1995 al 20/09/2012 a partir de la cual se consideraron las primeras cinco páginas de resultados para cada una de las palabras consideradas en el estudio, esto con el objetivo de observar a través de qué géneros discursivos y de qué grupos sociales se han expandido las palabras, así como el grado de integración tanto de los préstamos ya asentados como de los préstamos más recientes.

Por último, el tercer nivel de búsqueda consistió en un cuestionario realizado a doce personas. Para dicho cuestionario se consideraron las variables sociales de género, edad y grado de instrucción. ${ }^{8}$ El contenido del cuestionario se divide en tres partes; la primera contiene preguntas cuyo objetivo es averiguar si el informante ha escuchado la palabra en cuestión, si la utiliza y si sabe su significado. La segunda parte incluye preguntas que buscan dar cuenta de la expansión social de las unidades léxicas consideradas. Finalmente, la tercera parte es una prueba prescriptiva cuya finalidad es obtener información sobre la seguridad lingüística de los informantes. El interés principal de este cuestionario es contar con una pequeña comprobación de las reacciones de algunos hablantes del español mexicano a las tendencias que sugieren los otros dos niveles de búsqueda con respecto a las unidades léxicas estudiadas. Estamos

8 El género considera hombres $(\mathrm{H})$ y mujeres $(\mathrm{M})$. La edad comprende tres generaciones: la primera formada por personas de entre 20 y 34 años de edad (1); la segunda conformada por personas de entre 35 y 54 años (2) y la tercera comprende individuos de 55 años en adelante (3). El grado de instrucción se divide en alto (A) y bajo (B). El grado de instrucción alto considera informantes que por lo menos hayan terminado una licenciatura. El grado de instrucción bajo incluye informantes que como máximo hayan cursado la secundaria. Se cuenta entonces con 12 informantes, seis hombres y seis mujeres distribuidos en tres grupos de edad y dos grados de instrucción respectivamente. 
conscientes de que, para confirmar la trascendencia y validez de estas reacciones, lo ideal sería ampliar el número de hablantes por casilla para así contar con una muestra representativa; no obstante, las respuestas de los entrevistados ofrecen algunos indicios que bien valdría estudiar a profundidad. En consecuencia, por el momento, las respuestas de los informantes deben tomarse como meros indicios.

Por medio de estos tres niveles de búsqueda se ha querido conjuntar tanto datos de producción como datos de competencia para contrastarlos al servicio de la penetración y difusión de los préstamos. Se espera que los análisis que se muestran a continuación permitan un acercamiento al proceso de integración que han sufrido estas unidades léxicas en el español de México.

\section{ANÁlisis de DATOS}

\subsection{La palabra bullying}

El primer paso del análisis fue buscar las ocurrencias de la palabra bullying en los corpus mencionados en la sección anterior. La tabla 1 muestra los resultados de estas búsquedas.

Tabla 1. Número de ocurrencias de la palabra bullying en los distintos corpus consultados

\begin{tabular}{lccccl}
\hline Palabra & CEMC & CREA & CEMD & CORDE & CSCM \\
\hline bullying & 0 & 0 & 0 & 0 & Nivel alto: 0 \\
& & & & & Nivel medio: 0 \\
& & & & & Nivel bajo: 0 \\
\hline
\end{tabular}


La tabla 1 muestra que no se presentó ocurrencia alguna de la palabra bullying en los corpus que se consultaron. Esto puede interpretarse de dos maneras. Por un lado, se confirma que para realizar estudios sobre léxico no siempre resulta suficiente la consulta de corpus (Serrano 2011:191). Por el otro, se presta como un argumento para interpretar que el uso de la palabra bullying en el español de México es muy reciente como para aparecer documentada en este tipo de fuentes.

En cuanto a las búsquedas que se realizaron en Google, la tabla 2 muestra los resultados obtenidos durante la búsqueda por periodos de tiempo.

La tabla 2 advierte que existe un incremento exponencial de las ocurrencias de la palabra bullying considerando los tres periodos de tiempo en cuestión. En relación a lo anterior, si bien resulta cuestionable la exactitud de los resultados obtenidos, no deja de llamar la atención que el comportamiento de esta aparente tendencia se ajuste a la hipótesis de que la palabra bullying es un término que está en pleno momento de expansión. Ahora bien, si se toma en consideración que, de acuerdo con el INEGI, ${ }^{9}$ en el año 2000 había alrededor de 5.5 millones de usuarios de Internet en México, que para 2006 el número incrementó a 20.5 millones de usuarios y para 2011 se contabilizaron alrededor de 40.5 millones de usuarios, cabría la posibilidad de que el incremento en el empleo de la palabra se deba exclusivamente al incremento del número de usuarios. No obstante, si partimos de la premisa de que el uso de la palabra bullying en Internet tiene una relación proporcional al número de usuarios, resulta que el aumento exponencial en el uso supera al incremento estimado de usuarios

\footnotetext{
9 http://www.inegi.gob.mx/est/contenidos/espanol/temas/Sociodem/notatinf212.asp
} 
tal y como lo muestra la tabla 2. Esto sugiere que esta aparente difusión en el empleo de la palabra no se debe nada más a este factor.

Tabla 2. Índice de ocurrencias de la palabra bullying en Google en tres periodos de tiempo distintos

\begin{tabular}{|c|c|c|c|}
\hline Periodo & $\begin{array}{l}\text { Uso de la palabra } \\
\text { bullying }\end{array}$ & $\begin{array}{c}\text { N. de } \\
\text { usuarios de } \\
\text { internet }\end{array}$ & $\begin{array}{l}\text { Incremento de acuerdo } \\
\text { a la relación entre el uso } \\
\text { y el número de usuarios* }\end{array}$ \\
\hline $\begin{array}{l}01 / 01 / 1995- \\
31 / 12 / 2000\end{array}$ & Real: 729 resultados & $\begin{array}{r}5.5 \\
\text { millones }\end{array}$ & - \\
\hline $\begin{array}{l}01 / 01 / 2001- \\
31 / 12 / 2006\end{array}$ & $\begin{array}{l}\text { Estimado: } 2717 \text { resultados } \\
\text { Real: Cerca de } 4,450 \text { resultados }\end{array}$ & $\begin{array}{r}20.5 \\
\text { millones }\end{array}$ & $64 \%$ \\
\hline $\begin{array}{l}01 / 01 / 2007- \\
20 / 09 / 2012\end{array}$ & $\begin{array}{l}\text { Estimado: } 5368 \text { resultados } \\
\text { Real: Cerca de } 186000 \text { resultados. }\end{array}$ & $\begin{array}{r}40.5 \\
\text { millones }\end{array}$ & $3365 \%$ \\
\hline
\end{tabular}

* El cálculo se hizo por medio de una regla de tres donde se multiplicó el número de usuarios del segundo periodo de tiempo por el número de ocurrencias de la palabra en el primer periodo de tiempo dividido entre el número de usuarios del primer periodo de tiempo. Para el tercer periodo de tiempo, así como para el resto de los cálculos que se realizaron, se repitió el mismo procedimiento; es decir, siempre se hizo la comparación a partir del primer periodo considerado. Por medio de este primer cálculo, se obtuvo el estimado del número de ocurrencias de la palabra. Posteriormente, se determinó el incremento en el porcentaje con relación al número real de resultados obtenidos. Es decir, se obtuvo la diferencia entre el número real de resultados y el número estimado de ocurrencias del término divididas entre el estimado del periodo en cuestión para finalmente obtener el porcentaje de incremento con relación al número real de resultados obtenidos.

Otra cuestión a la que se prestó especial atención para darle mayor peso a nuestra argumentación fue la de observar si también se había presentado un incremento en el número de ocurrencias de unidades léxicas derivadas de la palabra bullying que se ajustaran a los procesos morfológicos del español. En relación a esto, la tabla 3 muestra el incremento en el uso tanto de formas alternas en las que se pensó podría aparecer la palabra bullying 
(bulling), como de formas derivadas y flexionadas que poco a poco se empiezan a escuchar entre los hablantes del español de México como buleado, buleador, bulear, buleando, entre otras.

Tabla 3. Ocurrencias de formas flexionadas y derivadas de la palabra bullying

\begin{tabular}{|c|c|c|c|}
\hline $\begin{array}{c}\text { Formas } \\
\text { gráfico-léxicas }\end{array}$ & $\begin{array}{c}01 / 01 / 1995- \\
31 / 12 / 2000\end{array}$ & $\begin{array}{l}01 / 01 / 2001- \\
31 / 12 / 2006\end{array}$ & $\begin{array}{c}01 / 01 / 2007- \\
20 / 9 / 2012\end{array}$ \\
\hline bulling & 10 resultados & Cerca de 357 resultados & Cerca de 20700 resultados \\
\hline bullear & 0 resultados & 0 resultados & 63 resultados \\
\hline bulear & 0 resultados & 1 resultado & 43 resultados \\
\hline bulleando & 0 resultados & 0 resultados & 27 resultados \\
\hline buleando & 0 resultados & 0 resultados & 4 resultados \\
\hline bulleable & 0 resultados & 0 resultados & 2 resultados \\
\hline buleable & 0 resultados & 0 resultados & 1 resultado \\
\hline buleado & 0 resultados & 0 resultados & 138 resultados \\
\hline buleado & 0 resultados & 3 resultados & 71 resultados \\
\hline buleador & 0 resultados & 2 resultados & 43 resultados \\
\hline bulleador & 0 resultados & 0 resultados & 22 resultados \\
\hline buleadores & 0 resultados & 1 resultado & 554 resultados \\
\hline bulleadores & 0 resultados & 0 resultados & 10 resultados \\
\hline
\end{tabular}

La tabla 3 respalda la hipótesis de que la unidad léxica bullying está en pleno proceso de expansión, ya que es a partir del periodo que va de 2007 a 2012 que encontramos un número relevante de ocurrencias de la palabra, así como de formas derivadas y alternancias ortográficas en los datos. Entre los procesos derivativos que se observan, destacan el sufijo verbalizante -ear y el sufijo nominalizador -dor. Los verbos denominales terminados en - ear se caracterizan por añadir dicho sufijo a la base sustantiva, elidiendo la 
vocal final cuando resulta necesario: bully > bulear; bat $(e)>$ batear; escáner > escanear; facebook > facebookear; faul > faulear; fumble > fumblear; hippy $>$ hippear etcétera. De acuerdo con Beniers (2010:97-98), las bases de este tipo de verbos pueden estar constituidas por préstamos, en su mayoría, del inglés, tal y como lo confirman los datos recién mencionados. En términos semánticos, el verbo bulear entra dentro de lo que Beniers (2010) caracteriza como un atributo comparativo de sujeto: 'hacer como un $X$ ', 'hacer de X' o 'comportarse como un X'. Dado lo anterior, la palabra bulear podría parafrasearse de la siguiente manera: bulear: 'hacer de acosador o bully'.

Por su parte, el sufijo -dor, se añade a una base verbal, previa elisión de la terminación final $-r$ de infinitivo, pero conservando la vocal temática del verbo en cuestión: batear > bateador; boxear > boxeador; bulear $>$ buleador; linchar >linchador; programar > programador etcétera. Este sufijo genera tanto a sustantivos como adjetivos a partir de verbos. En términos semánticos, Santiago Lacuesta y Bustos Gisbert (1999:4543-4544) mencionan que, cuando se trata de sustantivos animados, se pueden distinguir tres categorías distintas:

i. Los sustantivos clasificadores que suelen tener un significado con un sema de 'profesión' y pueden parafrasearse como 'alguien que profesionalmente hace $\mathrm{x}$ '.

ii. Los sustantivos caracterizadores, cuyo significado presenta un sema de 'persona caracterizada por su inclinación a realizar la acción designada por la base verbal'.

iii. Los sustantivos intensificadores que tienen un significado con un sema de 'persona que lleva a cabo la acción referida por el verbo o que la realizan en el momento de la enunciación'. 
Dada esta caracterización cabe parafrasear el significado de buleador como 'una persona que bulea a otra u otras'.

Como se ha venido señalando, los datos con los que se cuenta hasta el momento sugieren que es a partir del periodo de 2007/2012 cuando se ha comenzado a manifestar la adaptación lingüística del término bullying. A este respecto, resulta interesante observar que a nivel panhispánico el registro de la palabra bullying también es bastante reciente. De acuerdo con María Pozzi (2012):

En español, el Corpus de Referencia del Español Actual (CREA) de la Real Academia Española registra bullying por primera vez en 2000 y bully en 2002. En las Antenas Neológicas, el primer registro de ambos neologismos data de 2006: "Paula Vives es la actriz que encarna a Mercè Amorós, la estudiante víctima de bullying en la telenovela de TV-3, El cor de la ciutat. [El País, España, 02/06/2006]".

Ahora bien, en lo que respecta a los géneros discursivos en los que se registra el uso de la palabra bullying, de acuerdo con las primeras cinco páginas de resultados que se obtuvieron en el buscador Google (acotando la búsqueda a páginas de México y al periodo que va del 01/01/1995 al 20/09/2012), la palabra puede aparecer tanto en contextos formales como informales. A grandes rasgos, el término se registra en páginas que buscan informar qué es el bullying, revistas de interés general, blogs, periódicos, página especializadas en psicología, entre otras. En la imagen 1 se observan algunos de estos registros: 
Imagen 1. Ejemplo de páginas en las que se registra el uso de la palabra

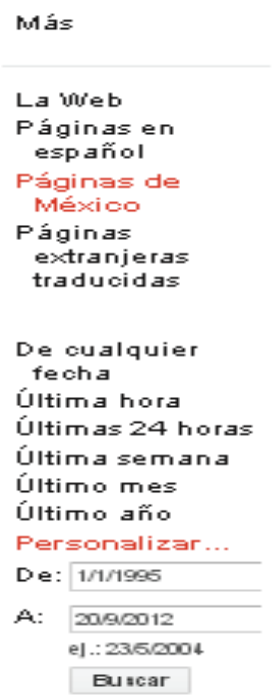

$$
\text { bullying }
$$

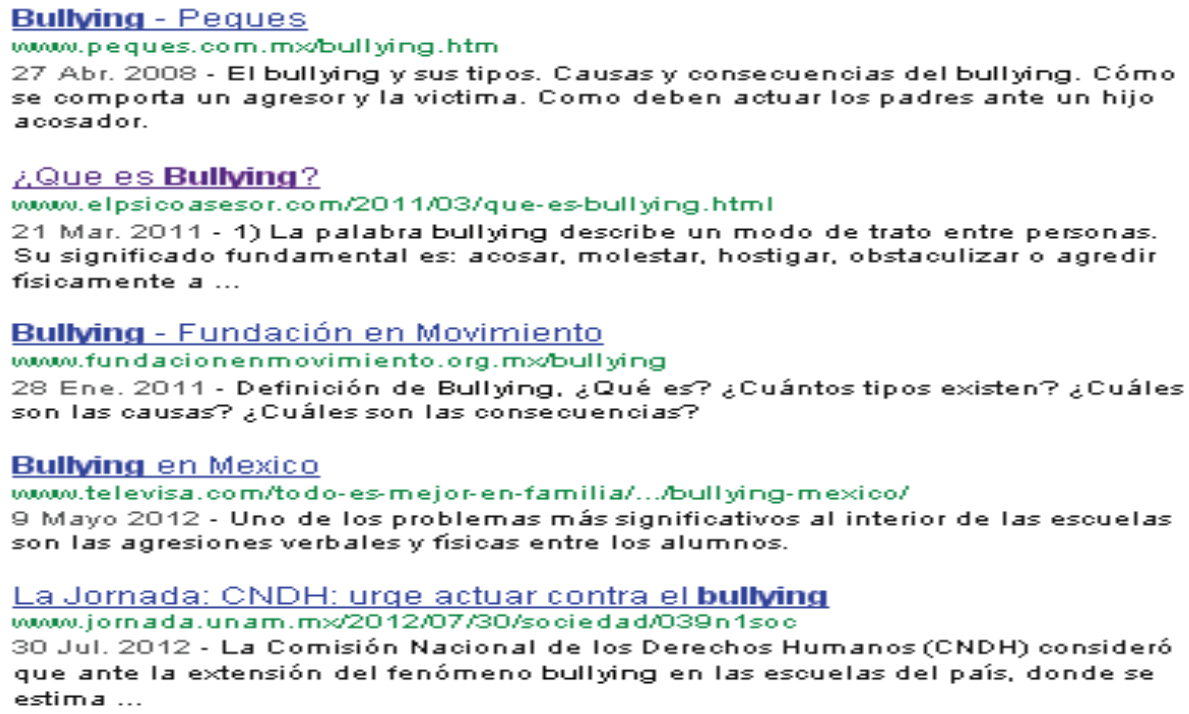

La imagen 1 ejemplifica el tipo de páginas en las que se registra el término bullying. El hecho de encontrar páginas en las que abiertamente se explica qué se debe entender por bullying y se describen sus principales características respalda la hipótesis de que se trata de un préstamo de reciente aparición, pues ejemplos como los que se muestran en la imagen 2 son un indicador de que el significado del término todavía no está del todo asentado y sigue en negociación.

\subsubsection{Datos del cuestionario ${ }^{10}$}

En cuanto a la información que proporciona el cuestionario realizado, la mayoría de los informantes reconocieron haber escuchado la palabra

10 Todas las tablas que contienen información relacionada con el cuestionario aparecen en el Anexo que se encuentra al final de este documento. 
Imagen 2. Negociación del significado de la palabra bullying

\begin{abstract}
Inicio > Todas las categoŕas > Salud y Belleza > Cuidados Generales de la Salud > Otros - Cuidados Generales

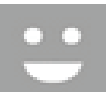

Alex?

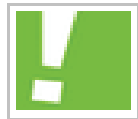

Donnie

Pregunta resuelta

\section{¿Qué es el Bullying en México?}

Me podrían decir que es exactamente ?? Que no sea información de wikipedia!? Bullying aqui en México ....

tace 1 alio

Mejor respuesta - elegida por los votantes

Violencia escolar...

El acoso escolar o 'bullying' es un problema que preocupa tanto a padres de familia como a escuelas. Causas y formas de manejarlo.

El bullying es un término cada vez más empleado, aunque no sea reciente su existencia, proviene del idioma holandés y significa acoso, se utiliza para nombrar al acoso que sufren los menores en la escuela. México no es una país exento de este fenómeno y la SEP ha comenzado a llevar a cabo programas para combatirlo.
\end{abstract}

bullying. De hecho, todas las personas con grado de instrucción alto mencionaron conocerla, y solo dos personas, ambas mujeres, perteneciente al grupo de grado de instrucción bajo, señalaron nunca haberla escuchado. Cabe preguntarse hasta qué punto influye el hecho de que estas personas tengan una relación estrecha con familiares que actualmente están cursando la primaria, la secundaria o la prepa, pues es de llamar la atención que incluso las personas del grupo de edad 3 y de grado de instrucción bajo aseguren haber escuchado esta palabra.

Ahora bien, resulta más interesante lo que dejan entrever los datos relacionados con la descripción del significado de la palabra bullying. Si contrastamos los datos obtenidos de los informantes con grado de instrucción alto con los de grado de instrucción bajo, se muestra revelador que 
los informantes con grado de instrucción alto parezcan tener una mejor idea del significado de la palabra. Algunos ejemplos de las respuestas obtenidas se muestran en (1):

(1) a. "Estar molestando a una persona o estar fastidiando a una persona. Normalmente en... se da en las escuelas." (HA1)

b. "El pasarte de lanza con tus compañeros (de escuela)." (MA1)

c. "Maltrato a las personas en las escuelas, también en el área en la que estoy porque hay bullying laboral." (HA3)

d. "Lo de las escuelas ¿no?, se agreden a los nińos." (MB3)

Al mismo tiempo, se debe mencionar que en la mayoría de los casos donde se obtuvo algún tipo de definición sobre el significado del término, el significado aparece relacionado con la escuela. Cabe preguntarse si el significado de la unidad léxica bullying terminará restringiéndose al ámbito escolar o si pasará a ser algo mucho más genérico; en este sentido, se debe averiguar si realmente se está gestando una competencia entre la palabra bullying y la palabra acosar ${ }^{11} \mathrm{y}$, en caso de que así sea, estudiar en qué condiciones se está dando. A este respecto, resulta interesante señalar que de acuerdo con María Pozzi (2012):

11 Marius Sala (1988:148) señala que en muchas ocasiones los préstamos aparecen sin que por ello se reemplace por completo el uso de palabras antiguas que se utilizan para designar situaciones o realidades similares. Por lo mismo, al menos durante un tiempo, tanto el préstamo como la palabra antigua pueden funcionar como sinónimos. 
La palabra bully es un préstamo del inglés, en donde según el Merriam Webster Learner's Dictionary (MW), funciona como sustantivo / adjetivo: 'a person who uses strength or power to harm or intimidate those who are weaker' $\mathrm{o}$ como verbo, con dos acepciones: 1) 'to frighten, hurt, or threaten (a smaller or weaker person): to act like a bully toward (someone)'; 2) 'to cause (someone) to do something by making threats or insults or by using force - usually + into'. El MW también registra bullying como sustantivo y proporciona el siguiente ejemplo: "bullying is not tolerated at school".

Según el Oxford English Dictionary, a finales del siglo xvı bully ya tenía un significado similar al actual: 'harasser of the weak'. El verbo to bully apareció alrededor de 1710 y posteriormente, bullying se nominalizó.

La definición de la palabra bullying en inglés no se restringe al ámbito escolar, por lo mismo, se debe tener en consideración que el significado de esta pieza léxica en los usos que la gente le está dando en el español de México parece circunscribirse, en un gran número de casos, al ámbito escolar.

Por otro lado, en lo que concierne al empleo de la palabra bullying por parte de los informantes, los datos obtenidos sugieren que en su mayoría son los jóvenes los que afirman utilizarla, mientras que los adultos se muestran más reticentes al respecto.

Como se mencionó en la introducción de este trabajo, la segunda parte del cuestionario consistió en realizar preguntas vinculadas con la expansión social de las palabras en cuestión. Sobre la palabra bullying, al preguntarles a los informantes sobre el tipo de gente que en su opinión usa dicho término, varias de las respuestas coinciden en mencionar que en padres de familia, hijos y jóvenes. Los datos que ejemplifican esto se presentan en (2): 
(2) Pregunta: ¿Qué tipo de personas utilizan la palabra bullying?

a. "Ahorita está muy de moda, creo que los papás... y ya si su hijo está siendo acosado o molestado en la escuela dicen: 'ah, ya tiene bullying' y es por influencia de los medios de comunicación.” (HA1)

b. "Los jóvenes, aunque la he escuchado mucho en CNN. La utilizan los adultos ya como un problema." (HA2)

c. "Las mamás, padres de familia, maestros." (MA2)

d. "Los jóvenes, es una palabra que ellos utilizan." (MA3)

e. "Los que están relacionados con esa palabra, esa moda. El padre con el hijo, el hijo con el papá." (HB1)

f. "Los padres que tienen hijos en las escuelas, son los que usan esa terminología malamente.” (HB3)

Algo que resulta llamativo es que tanto en ( $2 a)$ como en (2b) se expresa abiertamente el uso de la palabra en los medios de comunicación. Estas intuiciones de los informantes coinciden con los hallazgos de esta investigación que apuntan a pensar que podría tratarse de un cambio desde arriba, pues como señala Silva Corvalán (2001:245), los cambios desde arriba son cambios conscientes que se inician en los grupos sociales de mayor prestigio. Pareciera ser que con la palabra bullying se está generando un patrón valorado socialmente que con el apoyo y respaldo de los medios de comunicación comienza a imponerse entre los diferentes hablantes del país que poco a poco comienzan a adoptar el modelo impuesto desde arriba.

También se obtuvieron datos interesantes relacionados con la siguiente pregunta: 
(3) ¿En boca de quién se/te imagina(s) la palabra bullying?

De acuerdo con la información con la que contamos, existe una notoria tendencia en las respuestas de los informantes a imaginar la palabra bullying en boca de jóvenes y adultos. Esto también sugiere que la palabra está en pleno momento de expansión, pues de acuerdo con los informantes del primer y segundo grupo de edad, un adulto mayor difícilmente utilizaría la palabra bullying.

Por último, en relación a la prueba prescriptiva del cuestionario, las respuestas recibidas también revelan algunas cuestiones sugerentes. Las primeras preguntas de esta sección dirigen a los informantes a que escojan una respuesta entre dos o más opciones, como se muestra en (4) y (5):

(4) ¿Cómo llamaría(s) a alguien que practica el bullying? Por ejemplo, "Juan es un..."
a. bully
b. buleador
c. acosador

(5) ¿Cómo llamarías a alguien que sufre el bullying?
a. acosado
b. buleado

En términos generales, existe una marcada preferencia de los informantes por el uso de las unidades léxicas acosador y acosado, respectivamente. Solamente en el caso de los informantes del primer grupo de edad y de 
grado de instrucción alto se percibe una preferencia por utilizar formas como bully, buleador y buleado que, de acuerdo con la hipótesis de este trabajo, son de reciente aparición. Dados estos hallazgos, sería muy oportuno ahondar al respecto y verificar por medio de una muestra más amplia si otros informantes que entraran en estas categorías también se inclinarían por el uso de estas formas.

Asimismo, al preguntarles abiertamente a los informantes si consideraban que bullying está bien dicho en español, la respuesta que más se obtuvo fue la de no. Esto, incluso en aquellos hablantes del primer grupo de edad y de grado de instrucción alto, que indicaron preferir utilizar formas como bully o buleador a acosador. Finalmente, al preguntarles abiertamente si resulta preferible utilizar bullying en vez de acoso, los informantes, en su mayoría, se inclinaron por la unidad léxica acoso.

Es de llamar la atención que aquellos informantes que señalaron que bullying sí está bien dicho en español parecen considerar que existe cierta diferencia de significado entre acoso y bullying. Por lo mismo, los ejemplos que aparecen en (6) podrían justificar por qué desde la perspectiva de estos hablantes el uso de bullying es correcto:

(6) a. "Acoso yo lo siento como algo de sexual, yo diría algo más como agresión o agresión hacia los niños." (MB3)

b. "Acoso puede aplicarse a más cosas, el bullying solo a una golpiza o terror psicológico." (HA2)

c. "Bullying es botanearse a los chavos y acoso ya es excederse es algo más pesado. Son más pesadas las maldades." (HB1) 


\subsubsection{Síntesis}

Las tres dimensiones de búsqueda que se consideraron para dar cuenta del momento de expansión en que se encuentra el anglicismo bullying sugieren que se trata de un préstamo de reciente aparición. La nula ocurrencia de la palabra en los corpus considerados sugiere que estamos ante una pieza léxica que no está lo suficientemente asentada como para aparecer en este tipo de fuentes. A su vez, el hecho de que en Google la frecuencia de su uso se empiece a incrementar a partir del periodo que va de 2007 a 2012, así como la manifestación de formas derivadas en ese mismo lapso de tiempo, refuerzan la hipótesis de que el término está en pleno proceso de integración al español de México. De igual modo, la información que proporcionan las preguntas del cuestionario deja entrever que la palabra todavía no es reconocida por todos y que su significado todavía no es del todo claro y se sigue negociando. Por último, las respuestas de la parte prescriptiva del cuestionario manifiestan que a pesar de que hay un grupo mayoritario que no está de acuerdo con el uso del término bullying en lugar de acoso, los jóvenes parecen ser los más proclives a utilizarla, incluso si reconocen abiertamente que sería mejor optar por el empleo de la palabra acoso. En resumidas cuentas, se considera que todos estos indicadores son un buen punto de partida para seguir ahondando en el desarrollo del proceso de integración que está teniendo este anglicismo en el español de México.

\subsection{La palabra gay}

En cuanto a la palabra gay, la tabla 4 muestra los resultados de las búsquedas realizadas en los corpus seleccionados. 
Tabla 4. Número de ocurrencias de la palabra gay en los distintos corpus consultados

\begin{tabular}{lrrrrl}
\hline Palabra & CEMC & CREA & CEMD & CORDE & CSCM $^{*}$ \\
\hline gay & 0 & 18 & 1 & 0 & Nivel alto: 4 \\
& & & & & Nivel medio: 14 \\
& & & & & Nivel bajo: 3 \\
\hline
\end{tabular}

* Las cuatro ocurrencias de nivel alto se registraron en la misma entrevista, las doce de nivel medio en dos entrevistas distintas y las tres de nivel bajo también en la misma entrevista.

A diferencia de lo acontecido con la palabra bullying, la palabra gay sí se documentó en algunas de estas fuentes. Esto parece indicar que la palabra tiene un mayor grado de penetración que bullying. En particular, llama la atención que en el CSCM se registra la palabra en por lo menos en una de las entrevistas para cada grado de instrucción (7):

(7) a. “... pero luego nos dimos cuenta que es una playa de gays". (ME-10731M-00, nivel alto)

b. “... [hasta esa] fecha/ pero yo/ pues < - pus> yo/ sí me gustaba <- gusta:ba>/ pero pues < - pus > obviamente yo no sabía si él era gay < - guey> o no/ entonces/ pasó otra cosa con él/ é- él estuvo/ enamorado/ pero súper enamorado/ en aquel entonces/ de otro chavo del mismo ballet/..." (ME-271-21H-06, nivel medio)

c. "[y ahora] que ya lo llevo a los ensayos y eso/ ve que no/ no el hecho de ser gay <- guei>/ significa que bailes [clásico]" (ME-279-22H-06, nivel medio)

d. “...pues sí porque/ luego/ por decirlo así// una ocasión me tocó un gay/ pero haz de cuenta [que]...” (ME-291-11H-06, nivel bajo) 
En el CREA se documentaron un total de 18 casos en 12 textos distintos, donde la ocurrencia más antigua data de 1985 y la más reciente es del año 2003. Asimismo, resulta significativo observar que los géneros discursivos en los que más se documenta esta pieza léxica son textos narrativos de ficción y de interés general (arte y salud):

Tabla 5. Estadísticas del CREA sobre las ocurrencias de la palabra gay

\begin{tabular}{lccccclcc}
\hline Año & \% & Casos & País & \% & Casos & Tema & \% & Casos \\
\hline 1996 & 16.66 & 3 & México & 100.00 & 18 & 7.- Ficción. & 33.33 & 6 \\
1997 & 16.66 & 3 & & & & 4.- Artes. & 27.77 & 5 \\
2002 & 16.66 & 3 & & & & 6.- Salud. & 22.22 & 4 \\
2003 & 16.66 & 3 & & & & 2.-Ciencias sociales, & 11.11 & 2 \\
1995 & 11.11 & 2 & & & & creencias y & & \\
1985 & 5.55 & 1 & & & & pensamiento. & & \\
1987 & 5.55 & 1 & & & & 8.- Miscelánea. & 5.55 & 1 \\
1993 & 5.55 & 1 & & & & & & \\
1999 & 5.55 & 1 & & & & & & \\
\hline
\end{tabular}

Otra cuestión importante, es que, a diferencia de bullying, la palabra gay sí aparece en el DEM:

\section{gay}

s y adj $\mathrm{m}$ y $\mathrm{f}$ (Se pronuncia guéi) Persona homosexual, en particular la de sexo masculino: "Gays de todo el mundo hicieron una convención en Acapulco", "orgullo gay".

Por su parte, los resultados obtenidos a través de Google aparecen en la tabla 6: 
Tabla 6. Índice de ocurrencias de la palabra gay en Google en tres periodos de tiempo distintos

\begin{tabular}{|c|c|c|c|}
\hline Periodo & $\begin{array}{c}\text { Uso de la palabra } \\
\text { gay }\end{array}$ & $\begin{array}{c}\text { N. de } \\
\text { usuarios } \\
\text { de internet }\end{array}$ & $\begin{array}{l}\text { Incremento de acuerdo } \\
\text { a la relación entre el uso } \\
\text { y el número de usuarios }\end{array}$ \\
\hline $\begin{array}{l}01 / 01 / 1995- \\
31 / 12 / 2000\end{array}$ & Real: Cerca de 8370 resultados & $\begin{array}{r}5.5 \\
\text { millones }\end{array}$ & - \\
\hline $\begin{array}{l}01 / 01 / 2001- \\
31 / 12 / 2006\end{array}$ & $\begin{array}{l}\text { Estimado: } 31197 \text { resultados } \\
\text { Real: Cerca de } 91600 \text { resultados }\end{array}$ & $\begin{array}{r}20.5 \\
\text { millones }\end{array}$ & $193 \%$ \\
\hline $\begin{array}{l}01 / 01 / 2007- \\
20 / 09 / 2012\end{array}$ & $\begin{array}{l}\text { Estimado: } 61633 \text { resultados } \\
\text { Real: Cerca de } 333000 \text { resultados }\end{array}$ & $\begin{array}{r}40.5 \\
\text { millones }\end{array}$ & $440 \%$ \\
\hline
\end{tabular}

Como se puede observar, al igual que con la palabra bullying, se presenta un incremento exponencial de las ocurrencias de la pieza léxica gay a partir de los periodos de tiempo considerados. Sin embargo, si se comparan las ocurrencias para la palabra gay con las registradas para la palabra bullying, queda claro que las cifras estimadas para gay en los tres periodos en cuestión superan notoriamente las cifras obtenidas para la unidad léxica bullying. Esta información va de acuerdo con la hipótesis presentada al inicio de este trabajo de que el anglicismo gay tiene un mayor grado de difusión y distribución social por lo que se encuentra a la mitad del continuum propuesto.

Por otro lado, en lo que respecta a los géneros discursivos en los que aparece la pieza léxica gay en el buscador Google, observamos que la palabra tiene presencia tanto en contextos formales como informales. Por lo mismo, la palabra se documenta en foros de interés general, chats, páginas sobre destinos de viaje, revistas, periódicos y páginas exclusivamente 
creadas para hombres y mujeres homosexuales. La imagen 3, que aparece a continuación, ejemplifica algunos de estos registros:

Imagen 3. Ejemplo de páginas en las que se registra el uso de la palabra gay

\begin{tabular}{|c|c|}
\hline Más & Cine Gay Online \\
\hline & WW cinegayonline net/ \\
\hline La wieb & ginal We Once Were Tide AKA Una vez fuimos marea. Dirección Jason \\
\hline $\begin{array}{l}\text { Páginas en } \\
\text { español }\end{array}$ & ión Matthew Kyne Baskott Género Documental Pais RU ... \\
\hline $\begin{array}{l}\text { Páginas de } \\
\text { México }\end{array}$ & Gay | lugares de la Ciudad de México para encontrarte con tu ... \\
\hline $\begin{array}{l}\text { Páginas } \\
\text { extranjeras } \\
\text { traducidas }\end{array}$ & $\begin{array}{l}\text { Whw.timeoutmexico. } \mathrm{m} x / \mathrm{df} / \mathrm{gay} \\
\text { La sección enfocada al lector gay que también le encanta a los heterosexuales o } \\
\text { bugas. }\end{array}$ \\
\hline $\begin{array}{l}\text { De cualquier } \\
\text { fecha } \\
\text { Última hora } \\
\text { Últimas } 24 \text { hora: } \\
\text { Última semana } \\
\text { Último mes } \\
\text { Último an̂́o }\end{array}$ & $\begin{array}{l}\text { Noticias de gay } \\
\text { Celebran bodas gay en Washington } \\
\text { Decieros Televisa - hace } 11 \text { horas } \\
\text { Washington, luego de que las bodas gay fueran aprobadas el } 6 \text { de. }\end{array}$ \\
\hline Personalizar... & ata Oaxaca fallo sobre matrimonios gay. pero rechazará más solicitudes \\
\hline De: $1 / 1 / 1996$ & proceso,com. $\mathrm{mx}$ - hace 11 minutos \\
\hline A: 20102012 & Demócratas celebran revisión de la Corte sobre matrimonio gay \\
\hline $\begin{array}{c}\text { el.: } 2360004 \\
\text { Duscar }\end{array}$ & - hace 3 días \\
\hline
\end{tabular}

Si bien la palabra gay no muestra, por el momento, formas derivadas, sí se han generado algunas colocaciones y conceptos compuestos como orgullo gay, comunidad gay, bodas gay, matrimonio gay, antro gay, bar gay, desfile gay, baño gay entre otras.

\subsubsection{Datos del cuestionario}

En relación a la pregunta sobre el conocimiento de la palabra gay, todos los informantes aseguraron haberla escuchado antes, aspecto que refuerza 
la hipótesis de que la palabra tiene un mayor recorrido en el español mexicano que bullying. Asimismo, en cuanto a su significado, independientemente de cualquier variable social, todos los informantes dieron una definición bastante clara de lo que gay quiere decir: en la mayoría de los casos mencionaron la palabra homosexual para caracterizarla:

(8) Pregunta: ¿Qué quiere decir gay?
a. "Una persona homosexual." (HA1)
b. "Una persona que es homosexual." (MA2)
c. "Se utiliza para identificar a un grupo de homosexuales o una persona homosexual." (HA3)
d. "homosexual." (MA3)
e. "Son las personas que son homosexuales." (MB1)
f. "El término que usan para definir la homosexualidad de una per- sona.” (HB3)
g. "Son las personas que, cómo le dijera, son homosexuales." (MB3)

De acuerdo con el Merriam Webster Learner's Dictionary (MW), en inglés, la palabra gay se utiliza como sustantivo y como adjetivo. Como sustantivo, la palabra gay tiene un solo significado: 'a person and especially a man who is homosexual'; como adjetivo, la palabra gay presenta tres acepciones; dos de ellas de uso anticuado: 1) a. 'sexually attracted to someone who is the same sex: homosexual'; b. 'always used before a noun: of, relating to, or used by homosexuals'; 2) 'old-fashioned: happy and excited' o bien, 'cheerful and lively'; y 3) 'old-fashioned: very bright in color'. 
A partir de las respuestas en (8), así como de lo registrado en el DEM, queda claro que el campo de significaciones de la palabra gay en español está restringido al significado de la primera acepción que aparece en el MW cuando se usa como adjetivo, así como al único significado que tiene la palabra gay en inglés cuando se usa como sustantivo. Ahora bien, al preguntarles abiertamente a los informantes sobre la diferencia entre homosexual, gay y puto, 5 de los 12 informantes (3 de grado de instrucción bajo y 2 de grado de instrucción alto) señalaron que en su opinión las tres significan exactamente lo mismo, mientras que los demás consideraron que la palabra puto es más grosera, peyorativa o discriminatoria:

(9) Pregunta: ¿Cuál es la diferencia entre homosexual, gay y puto? ${ }^{12}$
a. "Ninguna, son sinónimos." (MA1)
b. "Ninguna, se me hace lo mismo." (HB1)
c. "Es lo mismo, solamente cambia la palabra." (HB2)
d. "Es lo mismo." (MB3)

12 Es importante tener en cuenta que de acuerdo con el DEM, en el español mexicano, la palabra puto cuenta con dos acepciones. Por un lado, la palabra como sustantivo masculino puede significar 'hombre homosexual', sin embargo, en otros contextos la palabra se puede utilizar como sustantivo o bien como adjetivo con el sentido de 'que es cobarde o miedoso'. Tomando esto en consideración, podría pensarse que el hecho de que la palabra puto tenga dos acepciones provoca que esta unidad léxica no esté exactamente en el mismo nivel de generalidad que las otras dos; no obstante, creemos que las preguntas del cuestionario como las respuestas ofrecidas por los informantes acotan y contextualizan el sentido de la palabra que estamos buscando, a saber, el de la primera acepción de la palabra según el DEM: 'hombre homosexual'. Vale la pena seguir indagando e investigar si la palabra gay en el español de México está adquiriendo el sentido de 'que es cobarde o miedoso' en algunos contextos. 
e. "Es lo mismo, aunque puto es más grosera." (MB1)

f. "Gay es una palabra en otro idioma, homosexual se usa en español para una persona que tiene preferencias sexuales del mismo sexo, y puto es más bien utilizada por el vulgo aunque quiere decir lo mismo.” (MA3)

g. "La palabra gay es darle cierta elegancia al término, homosexual se volvió una palabra tabú y puto ya es como una situación de discriminación." (HA3)

h. "Son sinónimos y simplemente es para degradar o poner más énfasis en su homosexualidad, puto sería una mala palabra." (MA2).

i. "Puto es muy despectivo y no me gusta en lo personal, gay y homosexual es el término para las personas que tienen estas preferencias." (HA2)

j. "Se oye mejor gay, es una palabra que no se oye tan fea, no se escucha tan drástica." (MB2)

En cuanto a si los hablantes utilizan o no la palabra gay, cinco de los seis informantes de grado de instrucción alto indicaron que sí la utilizan, mientras que tres de los seis informantes de grado de instrucción bajo señalaron que no. Si bien estos datos resultan interesantes, cabría indagar si las respuestas de los informantes se ajustan a la realidad y realmente el uso de la palabra gay está más difundido en aquellas personas que tienen un grado de instrucción alto.

Según nuestros datos, en general, los informantes imaginan la palabra gay en boca tanto de adolescentes, adultos y adultos mayores o en su defecto, en adolescentes y adultos. Estos indicadores parecen señalar que en la intuición de los hablantes el término gay parece estar bastante difundido. 
Finalmente, la prueba prescriptiva del cuestionario muestra que ante la pregunta sobre cómo debe decirse: Tengo muchos amigos que son: a) gay, b) gays o c) gayes, existe cierta variación entre los que consideran que la opción a) es la correcta y los que consideran que la opción b) es la que está bien. Ocho de los doce informantes indicaron que la opción b) era la correcta, mientras que el resto escogió la opción a). Si bien tres de los cuatro informantes que eligieron la opción a) como la correcta son mujeres, resulta arriesgado afirmar que esto signifique que haya cierta tendencia a que las mujeres utilicen la variante a). No obstante, se debe profundizar a este respecto para tener un panorama más claro en relación con esta variación.

Otro indicador que podría apoyar el argumento de que el uso de la palabra gay está más generalizado en aquellos individuos con un nivel de instrucción alto es que ante la pregunta de si consideran que gay está bien dicho en español, la mayoría de los del grupo con grado de instrucción alto señaló que sí (cuatro de seis), mientras que la mayoría de los del grupo con grado de instrucción bajo dijo que no (cuatro de seis). No obstante, también resulta indispensable contar con un mayor número de datos para poder afirmar o rechazar estos indicadores. Por último, ante la pregunta de si resulta preferible decir gay u homosexual, en términos generales, los individuos que pertenecen al primer y segundo grupos de edad muestran cierta preferencia por la pieza léxica gay, mientras que los que pertenecen al tercer grupo de edad prefieren el término homosexual. Esto resulta interesante ya que es a partir de finales de los años 70 cuando la comunidad gay en México comienza a aceptar abiertamente su homosexualidad en marchas y publicaciones. ${ }^{13}$ Este

13 ver http://www.letraese.org.mx/cronologia.htm 
tipo de eventos coincide con las fechas de nacimiento de los informantes que pertenecen al primer grupo de edad considerado (20-34 años). Por lo mismo, son los adolescentes y adultos jóvenes mexicanos de la actualidad los que han vivido más de cerca el proceso a través del cual la comunidad gay ha ido ganando más espacios sociales y aceptación en el país. Es probable que este tipo de acontecimientos influyan en la preferencia que muestran los informantes del primer y segundo grupo de edad por el anglicismo gay, pues la misma comunidad gay ha decidido autodenominarse con dicha palabra, aspecto que debe tomarse en cuenta.

\subsubsection{Síntesis}

Las tres dimensiones de búsqueda consideradas para aproximarnos a la historia de la expansión de la palabra gay sugieren que se trata de un préstamo con mucho mayor recorrido en la lengua que bullying. Por un lado, la ocurrencia de la palabra en algunos de los corpus consultados sugiere que se trata de una pieza léxica lo suficientemente usual como para aparecer en este tipo de fuentes. Por su parte, el hecho de que gay tenga una entrada en el DEM y que en Google la frecuencia de su uso se haga patente desde el primer periodo de tiempo considerado (01/01/1995-31/12/2000) son indicadores de que el término ya forma parte del español de México. De igual modo, la información que proporcionan las preguntas del cuestionario apuntan a que su significado está plenamente establecido; no obstante, parece ser que su uso se da sobre todo en jóvenes y adultos jóvenes. 


\subsection{La palabra checar}

La tabla 7 muestra las ocurrencias de checar en los corpus consultados.

Tabla 7. Número de ocurrencias de la palabra checar en los distintos corpus consultados

\begin{tabular}{lrrrrl}
\hline Palabra & CEMC & CREA & CEMD & CORDE & \multicolumn{1}{c}{ CSCM $^{*}$} \\
\hline checar & 17 & 42 & 7 & 1 & Nivel alto: 7 \\
& & & & & Nivel medio: 22 \\
& & & & & Nivel bajo: 9 \\
\hline
\end{tabular}

* Las siete ocurrencias de nivel alto se registraron en seis entrevista distintas, las 22 de nivel medio en nueve entrevistas distintas y las nueve de nivel bajo también en seis entrevistas distintas.

A diferencia de lo observado con la palabra gay, que apareció en solo algunos de los corpus consultados, la palabra checar se documenta por lo menos una vez en cada uno de los corpus considerados. Esto va de acuerdo con la hipótesis de que el anglicismo checar ya es una pieza léxica bien incorporado al español de México. Asimismo, resulta llamativo que en el CSCM se registra la palabra varias veces en distintas entrevistas para cada grado de instrucción:

(10) a. "... entonces les doy su clase/ me vengo corriendo/ checo los correos este// contesto/ trato de contestar siempre <-siempre: / lo que hay..." (ME-247-32M-05 571 I, nivel alto)

b. “... no/ entonces/ sí es es muy feo pero/ no todo es cáncer/tampoco o sea no/ no hablar de virus de papiloma humano es cáncer/ no// o 
sea hablar de virus de papiloma humano/ primero se debe de checar bien...” (ME-252-31M-05, nivel alto)

c. "...entonces $<-$ tos $>/$ pues $<-$ pus $>$ yo cada que tengo/ que estoy dando rondines/ tengo que checar/ ahora < - ora $>$ sí que los locales que estén ahí/ ya sean/ vinaterías// hay cantinas..." (ME-232-21H-04, nivel medio)

d. "este/ bueno/ el caso en cuestión del cine es eso no/ por eso/ yo estoy tan picado yo en/ en/ si veo una escena/ haz de cuenta/ nada < na> más ando checando/ si n- no no los errores/ porque no/ o sea no estoy cazando errores pero..." (ME-057-21H-99, nivel medio)

e. "...dice/ y/y le vamos a <decir> a la gente pues < - pus $>$ que/ vamos a checar bien el autobús y todo eso [para que no]..." (ME-130-12H-01, nivel bajo)

f. “...checando preguntándome que ¿qué ando haciendo?/ que < que:>/ qué es lo que hago ¿no?/ porque antes sí/ te digo que cuando deje de vender <de> este la droga..." (ME-304-11H-07, nivel bajo)

Por su parte, de las 42 ocurrencias de checar documentadas en el CREA, en la tabla 8 se muestra la fecha de aparición más antigua y más actual para cada una de las formas buscadas: ${ }^{14}$

14 Se buscaron las formas checar, checa, checó, chequé, checaba. 
Tabla 8. Ocurrencias documentadas en el CREA

\begin{tabular}{llrr}
\hline Forma & \multicolumn{1}{c}{ Número de ocurrencias } & Más antigua & Más reciente \\
\hline checar & 21 casos en 15 movimientos & 1977 & 2001 \\
checa & 4 casos en 4 documentos & 1980 & 1991 \\
checó & 3 casos en 3 movimientos & 1977 & 1997 \\
checando & 6 casos en 4 documentos & 1986 & 2001 \\
chequé & 3 casos en un solo documento & 186 & 1986 \\
checaba & 5 caos en 5 documentos distintos & 1996 & 2000 \\
Total & 42 casos en 31 documentos distintos & & \\
\hline
\end{tabular}

En la tabla 8 se muestra que el registro más antiguo de checar en el CREA data de 1977. Esto, aunado al hecho de que en el CEMC la ocurrencia más antigua de checar procede de 1963 (11), refuerza el argumento de que el término checar se encuentra en el extremo final del continuum propuesto:

(11) "Vino aquí a la escuela. y como le queda tan cerca, pues, aprovechó para llamarme y vino un ratito y se tenía que regresar a la scop porque tenía que checar su hora de salida pues no mucho, porque él en la tarde va a la universidad. y entonces muchas veces sale muy tarde de clase o tiene que entrar al..." (1963. Anónimo. Cinta 71-LII-A., CEMC)

Por su parte, la tabla 9 muestra los resultados de la búsqueda en Google. A partir de los resultados expuestos en la tabla 9, una vez más observamos que se presenta un incremento exponencial de las ocurrencias de la palabra checar a partir de los periodos considerados. No obstante, si bien se presentan más ocurrencias que las registradas para la palabra bullying, también es un hecho que para checar se registran menos ocurrencias que 
Tabla 9. Índice de ocurrencias de la palabra checar en Google en tres periodos de tiempo distintos

\begin{tabular}{|c|c|c|c|}
\hline Periodo & $\begin{array}{l}\text { Uso de la palabra } \\
\text { checar }\end{array}$ & $\begin{array}{c}\text { N. de } \\
\text { usuarios } \\
\text { de Internet }\end{array}$ & $\begin{array}{l}\text { Incremento de acuerdo a } \\
\text { la relación entre el uso y el } \\
\text { número de usuarios }\end{array}$ \\
\hline $\begin{array}{l}01 / 01 / 1995- \\
31 / 12 / 2000\end{array}$ & Real: Cerca de 1890 resultados & $\begin{array}{c}5.5 \\
\text { millones }\end{array}$ & - \\
\hline $\begin{array}{l}01 / 01 / 2001- \\
31 / 12 / 2006\end{array}$ & $\begin{array}{l}\text { Estimado: } 7044 \text { resultados } \\
\text { Real: Cerca de } 26800 \text { resultados }\end{array}$ & $\begin{array}{l}20.5 \\
\text { millones }\end{array}$ & $280 \%$ \\
\hline $\begin{array}{l}01 / 01 / 2007- \\
20 / 09 / 2012\end{array}$ & $\begin{array}{l}\text { Estimado: } 13917 \text { resultados } \\
\text { Real: Cerca de } 234000 \text { resultados }\end{array}$ & $\begin{array}{l}40.5 \\
\text { millones }\end{array}$ & $1581 \%$ \\
\hline
\end{tabular}

para gay. Esto no resulta contradictorio, ya que en la tabla 9 solamente se consideran las ocurrencias de checar en infinitivo; por ende, si tomáramos en cuenta todas las formas flexionadas del verbo checar, se registrarían muchas más ocurrencias que para el término gay. Para ejemplificar lo anterior, considérese la tabla 10:

Tabla 10. Ocurrencias de algunas formas flexionadas del verbo checar

\begin{tabular}{|c|c|c|c|}
\hline $\begin{array}{c}\text { FORMA } \\
\text { FLEXIONA- } \\
\text { DA }\end{array}$ & $\begin{array}{c}01 / 01 / 1995 \\
31 / 12 / 2000\end{array}$ & $01 / 01 / 2001-31 / 12 / 2006$ & $01 / 01 / 2007-20 / 09 / 2012$ \\
\hline checaste & 4 resultados & Cerca de 1140 resultados & Cerca de 56800 resultados \\
\hline chécate & 2 resultados & 373 resultados & Cerca de 56800 resultados \\
\hline checamos & 28 resultados & 617 resultados & Cerca de 30100 resultados \\
\hline chequen & 196 resultados & Cerca de 6130 resultados & Cerca de 173000 resultados \\
\hline checando & 125 resultados & Cerca de 3760 resultados & Cerca de 123000 resultados \\
\hline
\end{tabular}

Asimismo, los géneros discursivos en los que se documentó la palabra checar en el buscador Google engloban tanto contextos formales como 
informales. La pieza léxica aparece en páginas sobre cómo checar el aceite de un automóvil, checar un correo electrónico, páginas sobre consejos de distinta índole, periódicos, estados de crédito, foros, páginas de automóviles, motos, etcétera. La imagen 4 ilustra algunos de estos registros:

\author{
Imagen 4. Ejemplo de páginas en las que se registra el uso \\ de la palabra checar

La palabra checar también aparece documentada en el DEM con la siguientes dos acepciones:

\title{
checar
}

$\mathrm{v} \operatorname{tr}$ (Se conjuga como amar)

1 Comprobar la validez, la calidad o el buen estado de algo o alguien respecto de una norma, una lista o un catálogo previamente fijados: checar la presión del aceite, checar el aire de las llantas 
2 Marcar algo para comprobar alguna cosa como la entrada de alguien a un lugar, la utilización de algo, etc: checar los boletos, checar la tarjeta de asistencia

Con respecto a la segunda acepción, la palabra checar ha generado la forma derivada checador. De acuerdo con Lacuesta y Bustos Gisbert (1999), la unidades léxicas con el sufijo -dor que constituyen sustantivos inanimados, suelen tener un significado con semas de 'instrumento', 'lugar' o 'aparato' o 'máquina’. Dicho esto, la unidad léxica checador podría parafrasearse de la siguiente manera: 'máquina que se utiliza para checar la entrada y salida de alguien en algún lugar'.

Sobre la primera acepción, la palabra checar ha generado frases hechas como traer bien checadito a alguien y la forma derivada chequeo que ha dado lugar a la frase hecha hacerse un chequeo. De acuerdo con Lacuesta y Bustos Gisbert (1999:4584-4585), el sufijo -eo da forma a sustantivos deverbales ańadiéndose a verbos terminados en -ar y -ear; se trata de un sufijo que da lugar a derivados de género masculino que son "mayoritariamente 'nombres de acción'”. Dicho esto, la palabra chequeo podría parafrasearse como 'acción o efecto de checar'.

\subsubsection{Datos del cuestionario}

De acuerdo con el cuestionario realizado, todos los informantes reconocieron haber escuchado la palabra checar. De igual modo, en cuanto a su significado, todos los informantes dieron una definición vinculada con la primera acepción que aparece en el DEM; es importante señalar que en 
varios casos las palabras ver, mirar, observar y analizar aparecieron en las definiciones proporcionadas:

(12) Pregunta: ¿Qué quiere decir la palabra checar?

a. "...puede ser entre mira esto o pon atención a esto." (HA1)

b. "Observar." (MA1)

c. "Es como ver algo que esté bien o mal, una persona o un auto." (MA2)

d. "Observar o estar al pendiente de lo que hace alguien." (MB3)

e. "Revisar, observar, fijate en esto." (HB1)

f. "Es como analizar." (HB2)

Todos los informantes mencionaron que sí emplean la palabra checar. Esta información apoya la idea de que se trata de un préstamo totalmente acoplado al español mexicano. Por otro lado, ocho de los doce informantes señalaron que se imaginan la palabra en boca de cualquier tipo de persona, es decir, adolescentes, adultos y adultos mayores. Esto es otro indicador que muestra que la palabra está bien asentada en espańol mexicano.

En la prueba prescriptiva del cuestionario, ante la pregunta de si checar está bien dicho en español, solamente uno de los seis informantes con grado de instrucción alto dijo que no, mientras que cuatro de los seis informantes con grado de instrucción bajo consideraron que checar no está bien dicho en español. Esta información se complementa con la que se obtuvo por medio de la pregunta de si es preferible decir checar, verificar o ver, pues cuatro de los seis informantes con grado de instrucción alto señalaron que checar les parece perfectamente bien; mientras que cinco de los seis informantes con grado de instrucción bajo consideraron preferible utilizar alguna de las otras dos opciones. 


\subsubsection{Síntesis}

Las tres dimensiones de búsqueda realizadas para observar el nivel de expansión y penetración de la palabra checar sugieren que se trata de un préstamo que indudablemente ya forma parte del español mexicano. Por un lado, el hecho de encontrar registrada la palabra en todos los corpus consultados resulta un indicador claro de que la pieza léxica forma parte del vocabulario general de los usuarios; asimismo, las búsquedas en Google apoyan el argumento, ya que la palabra se puede encontrar en todo tipo de géneros discursivos. También, el hecho de encontrar formas derivadas, así como frases hechas que incluyen la unidad léxica checar, reflejan el grado de asentamiento que tiene la palabra entre los hablantes. Finalmente, la información proveniente del cuestionario permite dar cuenta de que el término es utilizado tanto por jóvenes como por adultos y adultos mayores. Quizá valdría la pena seguir investigando si realmente las personas con un grado de instrucción bajo consideran que, en lugar de checar, resulta preferible utilizar las unidades léxicas ver o verificar, tal y como sugieren los datos del cuestionario.

\section{Discusión}

$\mathrm{Al}$ inicio de este trabajo se planteó el objetivo de observar en qué momento de expansión se encuentran tres préstamos del inglés en el español de México. Por medio de los tres niveles de búsqueda considerados se ha querido dar una aproximación del grado de penetración y difusión que las palabras bullying, gay y checar tienen en la variante mexicana del español. 
Cuando consideramos que, a diferencia de otras unidades de la lengua como los fonemas o afijos, el léxico conforma un inventario abierto que se incrementa con facilidad dada su movilidad inherente, las limitaciones que conlleva trabajar tanto con corpus orales como escritos se magnifican. Es decir, además de lidiar con cuestiones generales como la autenticidad de los textos, el tipo de muestreo y representatividad (Tognini-Bonelli 2001:55-62), nos encontramos ante el hecho de que, en muchas ocasiones, no se registran ocurrencias de las unidades léxicas que estamos estudiando. Si a esto le ańadimos la inclinación por estudiar un área particular del léxico como es la de los anglicismos, la pesquisa de contextos de uso, así como de información relacionada con el tipo de personas y géneros discursivos en que estas piezas léxicas cobran vida queda todavía más restringida. Ante estas dificultades, buscar alternativas para la recolección de datos, como cuestionarios, entrevistas dirigidas y semidirigidas, búsquedas en Internet, etcétera, es una necesidad imperiosa.

En el caso particular de esta investigación, el empleo de una herramienta como el buscador de Google sirvió para documentar cuestiones relacionadas con el comportamiento morfológico de los anglicismos analizados, así como para indagar sobre su expansión en ciertos géneros discursivos. No obstante, se debe tener en cuenta que no todo aquello que aparece en Internet es fidedigno, razón por la cual se debe corroborar que efectivamente lo que encontramos corresponde tanto al fenómeno que estudiamos, como a la lengua o al dialecto que nos interesa. Por otro lado, si bien las búsquedas fechadas en Google permitieron dar cuenta de ciertos indicios sobre la expansión relativa de las piezas léxicas estudiadas, queda pendiente realizar una investigación más profunda y concisa que permita 
verificar si el incremento en el número de ocurrencias no se debe exclusivamente a que el volumen de información y la cantidad de usuarios de Internet en el territorio mexicano ha aumentado significativamente durante el último decenio.

En lo que respecta al cuestionario, a pesar de que en términos cualitativos las respuestas de los informantes revelan información valiosa relacionada con la estabilidad del significado de las palabras bullying, gay y checar, es indudable que para que estos datos adquieran verdadera representatividad y relevancia argumentativa se debe considerar un mayor número de informantes.

Lo que sí queda claro es que al intentar reconstruir el proceso de adaptación de estos préstamos al español de México, la noción de que cada palabra tiene su propia historia se manifiesta con mayor nitidez, pues la cantidad de información que se puede recolectar para cada pieza léxica siguiendo estas tres dimensiones de búsqueda es abundante. Esta observación plantea la posibilidad de concentrarse en una sola palabra, como bullying, para tratar a profundidad el tipo de géneros discursivos en el que una unidad léxica se va abriendo paso hasta volverse parte de la lengua general. Indiscutiblemente, esto implicaría implementar una metodología con criterios explícitos para obtener datos cualitativos y cuantitativos de los textos consultados para observar la pertinencia de hacer un trabajo de ese tipo.

De cualquier manera, como un primer ejercicio para acercarse al dinamismo del léxico de una lengua, utilizar buscadores como Google, con los criterios de búsqueda pertinentes, parece una buena herramienta para la recolección de datos. 


\section{Bibliografía}

Álvarez de Miranda, Pedro, 2004. "El léxico español, desde el siglo XviII hasta hoy". En Rafael Cano (coord.), Historia de la lengua española. Barcelona: Ariel, pp. 1037-1064.

Beniers Jacobs, Elisabeth, 2004. La formación de verbos en el español de México. México: El Colegio de México/Universidad Nacional Autónoma de México.

Curell, Clara, 2006. "La influencia del francés del español contemporáneo”. En Manuel Bruña Cuevas et al. (coord.), La cultura del otro: Español en Francia, francés en España. España: Universidad de Sevilla, pp. 785-792.

Figueroa, Manuel, 2003. "Cronología mínima del movimiento LGBT en México”. En Letra $S 83$ <www.letraese.org.mx/cronologia.htm.> [Consultado el 11 de diciembre de 2012]

Gómez Capuz, Juan, 1991. "Para una clasificación tipológica de los anglicismos en español actual”. En Julio Calvo Pérez (ed.), Lingüistica aplicada y tecnología. Actas del I Simposio. Valencia: Universidad de Valencia, pp. 63-70.

Gutiérrez Cortés, Fernando y Octavio Islas Carmona, 2008. "Historia del internet en México". En Hecho en C.U. <www.hechoencu.wordpress. com/2008/03/06/historia-de-la-Internet-en-mexico> [Consultado el 16 de noviembre de 2012]

Haugen, Einar, 1950. "The analysis of linguistic borrowing". Language 26 (2), pp. 210-231.

Humbley, John, 1974. "Vers une typologie de l'emprunt linguistique". Cahiers de Lexicologie 25 (2), pp. 46-70. 
Instituto Nacional de Estadística y Geografía, 2012. "Usuarios de Internet en México". En INEGI <www.inegi.gob.mx/est/contenidos/espanol/ temas/Sociodem/notatinf212.asp.> [Consultado el viernes 16 de noviembre de 2012]

Labov, Willian, 2010. Principles of Linguistic Change. Vol. 3: Cognitive and Cultural Factors. Oxford: Wiley-Blackwell, pp. 305-366.

Lacuesta, Ramón Santiago y Bustos Gisbert, Eugenio, 1999. "La derivación nominal”. En Ignacio Bosque y Violeta Demonte (dirs.), Gramática descriptiva de la lengua española, Vol 3. Madrid: Espasa Calpe, pp. 4505-4594.

Lapesa, Rafael, 1942. Historia de la lengua española. Madrid: Escelicer.

Lara, Luis Fernando, 1990. "El objeto diccionario". En Dimensiones de la lexicografía: A propósito del Diccionario del español actual de México. México: El Colegio de México, pp. 21-38.

Lastra Yolanda, y Pedro Martín Butragueńo (coords.) Corpus sociolingüistico de la Ciudad de México (CSCM). México: El Colegio de México. [véase http://lef.colmex.mx].

Lázaro Carreter, Fernando, 1987. "Los medios de comunicación y la lengua española". Primera reunión de Academias de la lengua española sobre el lenguaje y los medios de comunicación. Madrid: Real Academia Española, pp. 29-44.

Lorenzo, Emilio, 1996. Anglicismos hispánicos. Madrid: Gredos.

Mackey, William F. 1970. "Interference, integration, and the synchronic fallacy”. En James Alatis (ed.) Monograph Series on Language and Linguistics 23. Washington: Georgetown University Press, pp. 195-233. Meillet, Antoine, 1926. Linguistique historique et linguistique general I. París: Champion, pp. 230-271. 
Mejía, Hugo A., 1980. Préstamos de lenguas indígenas en el español americano del siglo XVII. México: Instituto de Investigaciones Filológicas, Universidad Nacional Autónoma de México.

Moreno de Alba, José G., 1992. "Anglicismos léxicos en España y América”. En Diferencias léxicas entre España y América. Madrid: Mampfre, pp. 195-230.

Office Québécois de la Langue Française, 2007. Politique de l'emprunt linguistique: Politique adoptée par l'Office Québécois de la Langue Française à sa séance du 14 septembre 2007. Québec: Office Québécois de la langue française. <www.oqlf.gouv.qc.ca/ressources/bibliotheque/terminologie/ Pol_empruntling_20070914> [Consultado el 25 de julio de 2014].

Otaola Olano, Concepción, 2004. Lexicología y semántica léxica. Teoría y aplicación a la lengua española. Madrid: Ediciones Académicas.

Pratt, Chris, 1980. El anglicismo en el español peninsular contemporáneo. Madrid: Gredos.

Pozzi, María, 2012. "Bullying". En Antenas Neológicas <www.iula.upf.edu/ rec/antenas/2012.04.neo_esp.htm.> [Consultado el 16 de noviembre de 2012].

Sala, Marius, 1988. El problema de las lenguas en contacto. México: Universidad Nacional Autónoma de México.

Serrano, Julio César, 2011. "Retracción e innovación léxica en el español de la ciudad de México, 1970-2000”. En Pedro Martín Butragueño (ed.), Realismo en el análisis de corpus orales. Primer coloquio de cambio y variación lingüistica. México: El Colegio de México, pp. 191-213.

Schifko, Peter, 1992. "Lexicología y semántica”. Lexikon der Romanistischen Linguistik VI (1), pp. 132-148. 
Silva-Corvalán, Carmen, 2001. Sociolingüistica y pragmática del español. Washington, D. C.: Georgetown University Press.

Sperber, Hans, 1923. Einführung in die Bedeutungslehere. Bonn/Leipzig: Schroeder.

Tognini-Bonelli, Elena, 2001. Corpus Linguistics at Work. Amsterdam-Filadelfia: John Benjamins.

Ullmann, Stephen, 1965. Semántica. Introducción a la ciencia del significado. Madrid: Aguilar

\section{Corpus}

Davies, Mark, 2002. Corpus del Español. Brigham Young University, en http://www.corpusdelespanol.org. [Consultado en octubre de 2012]. Diccionario del Español de México (DEM) <http: //dem.colmex.mx>, El Colegio de México, A.C. [Consultado en diciembre de 2012].

Diccionario del Español de México. Corpus del Español Mexicano Contemporáneo (CEMC). <http://www.corpus.unam.mx/cemc>, software AMATE ver. 1.0. [Consultado en octubre de 2012].

Merriam Webster Learner's Dictionary, s.v., "gay" <www.learnersdictionary. com> [Consultado en octubre de 2012].

Real Academia Española. Banco de datos (CREA) [en línea]. Corpus de referencia del español actual.<http://www.rae.es>. [Consulado en octubre de 2012].

Real Academia Española: Banco de datos (CORDE) [en línea]. Corpus diacrónico del español.<http://www.rae.es>. [Consultado en octubre de 2012]. 
ApÉNdice. Tablas CON los datos ObTenidos en el CUESTiOnario

Tabla I. Respuestas obtenidas en relación al conocimiento y desconocimiento de las palabras bullying, gay y checar

Respuesta: $s=$ sí, $n=n o$; Edad: $1=20-34$ años, $2=35-54$ años, $3=55$ años $\mathrm{en}$ adelante; Género: $H=$ hombre, $M=$ mujer; Nivel educativo: $A=$ alto, $B=$ bajo

\begin{tabular}{|c|c|c|c|}
\hline \multicolumn{4}{|c|}{ Pregunta: ¿ $\mathrm{Ha}(s)$ escuchado la palabra bullying? } \\
\hline Respuesta & Edad & Género & Nivel educativo \\
\hline $\mathrm{s}$ & 1 & $\mathrm{H}$ & A \\
\hline s & 1 & M & A \\
\hline s & 2 & $\mathrm{H}$ & A \\
\hline s & 2 & M & A \\
\hline s & 3 & $\mathrm{H}$ & A \\
\hline s & 3 & M & A \\
\hline$s$ & 1 & $\mathrm{H}$ & B \\
\hline $\mathrm{n}$ & 1 & M & B \\
\hline s & 2 & $\mathrm{H}$ & B \\
\hline $\mathrm{n}$ & 2 & M & B \\
\hline s & 3 & $\mathrm{H}$ & B \\
\hline s & 3 & M & B \\
\hline \multicolumn{4}{|c|}{ Pregunta: ¿ $\mathrm{Ha}(\mathrm{s})$ escuchado la palabra gay? } \\
\hline Respuesta & Edad & Género & Nivel educativo \\
\hline $\mathrm{s}$ & 1 & $\mathrm{H}$ & A \\
\hline s & 1 & M & A \\
\hline s & 2 & $\mathrm{H}$ & A \\
\hline s & 2 & M & A \\
\hline s & 3 & $\mathrm{H}$ & A \\
\hline s & 3 & $\mathrm{M}$ & A \\
\hline$s$ & 1 & $\mathrm{H}$ & B \\
\hline
\end{tabular}




\begin{tabular}{|c|c|c|c|}
\hline \multicolumn{4}{|c|}{ Pregunta: ¿Ha(s) escuchado la palabra gay? } \\
\hline Respuesta & Edad & Género & Nivel educativo \\
\hline$s$ & 1 & M & $\mathrm{B}$ \\
\hline$s$ & 2 & $\mathrm{H}$ & $\mathrm{B}$ \\
\hline$s$ & 2 & M & $\mathrm{B}$ \\
\hline$s$ & 3 & $\mathrm{H}$ & $\mathrm{B}$ \\
\hline$s$ & 3 & M & $\mathrm{B}$ \\
\hline \multicolumn{4}{|c|}{ Pregunta: ¿Ha(s) escuchado la palabra checar? } \\
\hline Respuesta & Edad & Género & Nivel educativo \\
\hline s & 1 & $\mathrm{H}$ & A \\
\hline$s$ & 1 & M & A \\
\hline$s$ & 2 & $\mathrm{H}$ & A \\
\hline$s$ & 2 & M & $A$ \\
\hline$s$ & 3 & $\mathrm{H}$ & $A$ \\
\hline s & 3 & $\mathrm{M}$ & $A$ \\
\hline$s$ & 1 & $\mathrm{H}$ & B \\
\hline$s$ & 1 & $\mathrm{M}$ & B \\
\hline s & 2 & $\mathrm{H}$ & B \\
\hline$s$ & 2 & $\mathrm{M}$ & B \\
\hline s & 3 & $\mathrm{H}$ & B \\
\hline$s$ & 3 & M & $\mathrm{B}$ \\
\hline
\end{tabular}


Tabla II. Conocimiento del significado de las palabras bullying, gay y checar

\begin{tabular}{|c|c|c|c|}
\hline \multicolumn{4}{|c|}{ Pregunta: ¿Qué quiere decir bullying? } \\
\hline $\begin{array}{l}\text { Índice de conocimiento } \\
\text { del significado }\end{array}$ & Edad & Género & Nivel educativo \\
\hline III & 1 & $\mathrm{H}$ & A \\
\hline III & 1 & M & A \\
\hline III & 2 & $\mathrm{H}$ & A \\
\hline II & 2 & M & A \\
\hline III & 3 & $\mathrm{H}$ & A \\
\hline II & 3 & M & A \\
\hline III & 1 & $\mathrm{H}$ & B \\
\hline 0 & 1 & M & B \\
\hline I & 2 & $\mathrm{H}$ & B \\
\hline 0 & 2 & M & B \\
\hline II & 3 & $\mathrm{H}$ & B \\
\hline II & 3 & M & B \\
\hline \multicolumn{4}{|c|}{ Pregunta: ¿Qué quiere decir gay? } \\
\hline $\begin{array}{c}\text { Índice de conocimiento } \\
\text { del significado }\end{array}$ & Edad & Género & Nivel educativo \\
\hline III & 1 & $\mathrm{H}$ & A \\
\hline III & 1 & M & A \\
\hline III & 2 & $\mathrm{H}$ & A \\
\hline III & 2 & M & A \\
\hline III & 3 & $\mathrm{H}$ & A \\
\hline III & 3 & M & A \\
\hline III & 1 & $\mathrm{H}$ & B \\
\hline III & 1 & M & B \\
\hline II & 2 & $\mathrm{H}$ & B \\
\hline III & 2 & M & B \\
\hline III & 3 & $\mathrm{H}$ & B \\
\hline III & 3 & $\mathrm{M}$ & B \\
\hline
\end{tabular}




\begin{tabular}{cccc}
\hline $\begin{array}{c}\text { Pregunta: ¿Qué quiere decir checar? } \\
\text { Indice de conocimiento } \\
\text { del significado }\end{array}$ & Edad & Género & Nivel educativo \\
\hline III & 1 & H & A \\
III & 1 & M & A \\
III & 2 & H & A \\
III & 2 & M & A \\
III & 3 & H & A \\
III & 3 & M & A \\
III & 1 & H & B \\
III & 1 & M & B \\
III & 2 & H & B \\
III & 2 & M & B \\
III & 3 & H & B \\
III & 3 & M & B \\
\hline
\end{tabular}

Tabla III. Uso de las palabras bullying, gay y checar Respuesta: $O=$ no la conoce, $s=s i, n=$ no

\begin{tabular}{cccc}
\hline Respuesta & $\begin{array}{c}\text { Pregunta: ¿Usted/Tú usa(s) la palabra bullying? } \\
\text { Edad }\end{array}$ & Género & Nivel educativo \\
\hline s & 1 & $\mathrm{H}$ & $\mathrm{A}$ \\
$\mathrm{s}$ & 1 & $\mathrm{M}$ & $\mathrm{A}$ \\
$\mathrm{n}$ & 2 & $\mathrm{H}$ & $\mathrm{A}$ \\
$\mathrm{n}$ & 2 & $\mathrm{M}$ & $\mathrm{A}$ \\
$\mathrm{s}$ & 3 & $\mathrm{H}$ & $\mathrm{A}$ \\
$\mathrm{n}$ & 3 & $\mathrm{M}$ & $\mathrm{A}$ \\
$\mathrm{s}$ & 1 & $\mathrm{H}$ & $\mathrm{B}$ \\
0 & 1 & $\mathrm{M}$ & $\mathrm{B}$ \\
$\mathrm{s}$ & 2 & $\mathrm{H}$ & $\mathrm{B}$ \\
0 & 2 & $\mathrm{M}$ & $\mathrm{B}$ \\
$\mathrm{n}$ & 3 & $\mathrm{H}$ & $\mathrm{B}$ \\
$\mathrm{n}$ & 3 & $\mathrm{M}$ & $\mathrm{B}$ \\
\hline
\end{tabular}




\begin{tabular}{|c|c|c|c|}
\hline \multicolumn{4}{|c|}{ Pregunta: ¿Usted/Tú usa(s) la palabra gay? } \\
\hline Respuesta & Edad & Género & Nivel educativo \\
\hline$s$ & 1 & $\mathrm{H}$ & $\mathrm{A}$ \\
\hline s & 1 & $\mathrm{M}$ & A \\
\hline$s$ & 2 & $\mathrm{H}$ & A \\
\hline s & 2 & M & A \\
\hline s & 3 & $\mathrm{H}$ & A \\
\hline $\mathrm{n}$ & 3 & $\mathrm{M}$ & A \\
\hline$s$ & 1 & $\mathrm{H}$ & $\mathrm{B}$ \\
\hline $\mathrm{n}$ & 1 & M & $\mathrm{B}$ \\
\hline $\mathrm{n}$ & 2 & $\mathrm{H}$ & $\mathrm{B}$ \\
\hline $\mathrm{n}$ & 2 & M & $\mathrm{B}$ \\
\hline s & 3 & $\mathrm{H}$ & $\mathrm{B}$ \\
\hline s & 3 & M & $\mathrm{B}$ \\
\hline \multicolumn{4}{|c|}{ Pregunta: ¿Usted/Tú usa(s) la palabra checar? } \\
\hline Respuesta & Edad & Género & Nivel educativo \\
\hline$s$ & 1 & $\mathrm{H}$ & A \\
\hline$s$ & 1 & M & A \\
\hline s & 2 & $\mathrm{H}$ & A \\
\hline$s$ & 2 & $\mathrm{M}$ & $\mathrm{A}$ \\
\hline$s$ & 3 & $\mathrm{H}$ & $\mathrm{A}$ \\
\hline$s$ & 3 & $\mathrm{M}$ & $\mathrm{A}$ \\
\hline s & 1 & $\mathrm{H}$ & B \\
\hline$s$ & 1 & $\mathrm{M}$ & B \\
\hline$s$ & 2 & $\mathrm{H}$ & B \\
\hline$s$ & 2 & $\mathrm{M}$ & B \\
\hline s & 3 & $\mathrm{H}$ & B \\
\hline $\mathrm{n}$ & 3 & $\mathrm{M}$ & B \\
\hline
\end{tabular}


Tabla IV. Respuestas: $0=$ no conoce la palabra, $a=$ adolescentes, $b=$ adultos,

$$
c=\text { adultos mayores }
$$

\begin{tabular}{|c|c|c|c|}
\hline \multicolumn{4}{|c|}{ Pregunta: ¿En boca de quién se/te imagina(s) la palabra bullying? } \\
\hline Respuesta & Edad & Género & Nivel educativo \\
\hline$a, b$ & 1 & $\mathrm{H}$ & A \\
\hline $\mathrm{b}$ & 1 & $\mathrm{M}$ & A \\
\hline$a, b$ & 2 & $\mathrm{H}$ & A \\
\hline $\mathrm{a}$ & 2 & $\mathrm{M}$ & A \\
\hline$a, b, c$ & 3 & $\mathrm{H}$ & A \\
\hline $\mathrm{a}$ & 3 & $\mathrm{M}$ & A \\
\hline $\mathrm{a}, \mathrm{b}$ & 1 & $\mathrm{H}$ & $\mathrm{B}$ \\
\hline 0 & 1 & M & B \\
\hline a & 2 & $\mathrm{H}$ & $\mathrm{B}$ \\
\hline 0 & 2 & M & B \\
\hline$a, b$ & 3 & $\mathrm{H}$ & B \\
\hline $\mathrm{b}, \mathrm{c}$ & 3 & $\mathrm{M}$ & B \\
\hline \multicolumn{4}{|c|}{ Pregunta: ¿En boca de quién se/te imagina(s) la palabra gay? } \\
\hline Respuesta & Edad & Género & Nivel educativo \\
\hline $\mathrm{a}, \mathrm{b}, \mathrm{c}$ & 1 & $\mathrm{H}$ & A \\
\hline$a, b, c$ & 1 & $\mathrm{M}$ & A \\
\hline$a, b, c$ & 2 & $\mathrm{H}$ & A \\
\hline $\mathrm{a}, \mathrm{b}$ & 2 & M & A \\
\hline$a, b, c$ & 3 & $\mathrm{H}$ & A \\
\hline $\mathrm{a}, \mathrm{b}$ & 3 & M & A \\
\hline$a, b, c$ & 1 & $\mathrm{H}$ & B \\
\hline $\mathrm{a}$ & 1 & $\mathrm{M}$ & B \\
\hline$a, b$ & 2 & $\mathrm{H}$ & B \\
\hline $\mathrm{a}, \mathrm{b}$ & 2 & $\mathrm{M}$ & B \\
\hline$a, b, c$ & 3 & $\mathrm{H}$ & B \\
\hline$a, b$ & 3 & $\mathrm{M}$ & $\mathrm{B}$ \\
\hline
\end{tabular}




\begin{tabular}{cccc}
\hline \multicolumn{3}{c}{ Pregunta: ¿En boca de quién se/te imagina(s) la palabra checar? } \\
Respuesta & Edad & Género & Nivel educativo \\
\hline a, b, c & 1 & $\mathrm{H}$ & $\mathrm{A}$ \\
b & 1 & $\mathrm{M}$ & $\mathrm{A}$ \\
a, b, c & 2 & $\mathrm{H}$ & $\mathrm{A}$ \\
a, b, c & 2 & $\mathrm{M}$ & $\mathrm{A}$ \\
$\mathrm{b}$ & 3 & $\mathrm{H}$ & $\mathrm{A}$ \\
a, b, c & 3 & $\mathrm{M}$ & $\mathrm{A}$ \\
$\mathrm{a}$ & 1 & $\mathrm{H}$ & $\mathrm{B}$ \\
$\mathrm{b}$ & 1 & $\mathrm{M}$ & $\mathrm{B}$ \\
a, b, c & 2 & $\mathrm{H}$ & $\mathrm{B}$ \\
a, b, c & 2 & $\mathrm{M}$ & $\mathrm{B}$ \\
a, b, c & 3 & $\mathrm{H}$ & $\mathrm{B}$ \\
a, b, c & 3 & $\mathrm{M}$ & $\mathrm{B}$ \\
\hline
\end{tabular}

Tabla V. Respuestas: $0=$ no conoce la palabra, $a=b u l l y, b=$ buleador, $c=$ acosador; $O=$ no conoce la palabra, $a=$ gay, $b=$ gayes, $c=$ gayes

¿Cómo llamaría(s) a alguien que practica el bullying?

Ej. Juan es un:

a) bully, b) buleador, c) acosador

\begin{tabular}{cccc} 
Respuesta & Edad & Género & Nivel educativo \\
\hline a & 1 & $\mathrm{H}$ & $\mathrm{A}$ \\
$\mathrm{b}$ & 1 & $\mathrm{M}$ & $\mathrm{A}$ \\
$\mathrm{c}$ & 2 & $\mathrm{H}$ & $\mathrm{A}$ \\
$\mathrm{c}$ & 2 & $\mathrm{M}$ & $\mathrm{A}$ \\
$\mathrm{c}$ & 3 & $\mathrm{H}$ & $\mathrm{A}$ \\
$\mathrm{c}$ & 3 & $\mathrm{M}$ & $\mathrm{A}$ \\
$\mathrm{a}$ & 1 & $\mathrm{H}$ & $\mathrm{B}$ \\
0 & 1 & $\mathrm{M}$ & $\mathrm{B}$ \\
$\mathrm{c}$ & 2 & $\mathrm{H}$ & $\mathrm{B}$ \\
0 & 2 & $\mathrm{M}$ & $\mathrm{B}$ \\
$\mathrm{c}$ & 3 & $\mathrm{H}$ & $\mathrm{B}$ \\
$\mathrm{a}$ & 3 & $\mathrm{M}$ & $\mathrm{B}$ \\
\hline
\end{tabular}


¿En su/tu opinión cómo debe decirse?

Ej. Tengo amigos que son: a) gay, b) gays c) gayes

\begin{tabular}{cccc} 
Respuesta & Edad & Género & Nivel educativo \\
\hline $\mathrm{b}$ & 1 & $\mathrm{H}$ & $\mathrm{A}$ \\
$\mathrm{a}$ & 1 & $\mathrm{M}$ & $\mathrm{A}$ \\
$\mathrm{b}$ & 2 & $\mathrm{H}$ & $\mathrm{A}$ \\
$\mathrm{a}$ & 2 & $\mathrm{M}$ & $\mathrm{A}$ \\
$\mathrm{b}$ & 3 & $\mathrm{H}$ & $\mathrm{A}$ \\
$\mathrm{b}$ & 3 & $\mathrm{M}$ & $\mathrm{A}$ \\
$\mathrm{b}$ & 1 & $\mathrm{H}$ & $\mathrm{B}$ \\
$\mathrm{b}$ & 1 & $\mathrm{M}$ & $\mathrm{B}$ \\
$\mathrm{b}$ & 2 & $\mathrm{H}$ & $\mathrm{B}$ \\
$\mathrm{a}$ & 2 & $\mathrm{M}$ & $\mathrm{B}$ \\
$\mathrm{a}$ & 3 & $\mathrm{H}$ & $\mathrm{B}$ \\
$\mathrm{b}$ & 3 & $\mathrm{M}$ & $\mathrm{B}$ \\
\hline
\end{tabular}

Tabla VI. Respuestas: $O=$ no conoce la palabra, $a=a \cos a d o, b=b u l e a d o$

Preguntas: ¿Cómo llamarías a alguien que sufre el bullying?

a) acosado, b) buleado

\begin{tabular}{cccc} 
Respuesta & Edad & Género & Nivel educativo \\
\hline $\mathrm{b}$ & 1 & $\mathrm{H}$ & $\mathrm{A}$ \\
$\mathrm{b}$ & 1 & $\mathrm{M}$ & $\mathrm{A}$ \\
$\mathrm{a}$ & 2 & $\mathrm{H}$ & $\mathrm{A}$ \\
$\mathrm{a}$ & 2 & $\mathrm{M}$ & $\mathrm{A}$ \\
$\mathrm{a}$ & 3 & $\mathrm{H}$ & $\mathrm{A}$ \\
$\mathrm{a}$ & 3 & $\mathrm{M}$ & $\mathrm{A}$ \\
$\mathrm{a}$ & 1 & $\mathrm{H}$ & $\mathrm{B}$ \\
0 & 1 & $\mathrm{M}$ & $\mathrm{B}$ \\
$\mathrm{a}$ & 2 & $\mathrm{H}$ & $\mathrm{B}$ \\
0 & 2 & $\mathrm{M}$ & $\mathrm{B}$ \\
$\mathrm{a}$ & 3 & $\mathrm{H}$ & $\mathrm{B}$ \\
$\mathrm{a}$ & 3 & $\mathrm{M}$ & $\mathrm{B}$ \\
\hline
\end{tabular}


Tabla VII. $O=$ no conoce la palabra, $s=$ si, $n=n o$

\begin{tabular}{|c|c|c|c|}
\hline \multicolumn{4}{|c|}{ Pregunta: ¿Usted/tú cree(s) que bullying está bien dicho en espańol? } \\
\hline Respuesta & Edad & Género & Nivel educativo \\
\hline $\mathrm{n}$ & 1 & $\mathrm{H}$ & A \\
\hline $\mathrm{n}$ & 1 & M & A \\
\hline $\mathrm{n}$ & 2 & $\mathrm{H}$ & A \\
\hline $\mathrm{n}$ & 2 & M & A \\
\hline$s$ & 3 & $\mathrm{H}$ & A \\
\hline $\mathrm{n}$ & 3 & $\mathrm{M}$ & A \\
\hline $\mathrm{n}$ & 1 & $\mathrm{H}$ & B \\
\hline 0 & 1 & $\mathrm{M}$ & B \\
\hline $\mathrm{n}$ & 2 & $\mathrm{H}$ & B \\
\hline 0 & 2 & $\mathrm{M}$ & B \\
\hline $\mathrm{n}$ & 3 & $\mathrm{H}$ & B \\
\hline $\mathrm{n}$ & 3 & $\mathrm{M}$ & B \\
\hline \multicolumn{4}{|c|}{ Pregunta: ¿Usted/tú cree(s) que gay está bien dicho en español? } \\
\hline Respuesta & Edad & Género & Nivel educativo \\
\hline$s$ & 1 & $\mathrm{H}$ & A \\
\hline$s$ & 1 & $\mathrm{M}$ & A \\
\hline$s$ & 2 & $\mathrm{H}$ & A \\
\hline $\mathrm{n}$ & 2 & $\mathrm{M}$ & A \\
\hline$s$ & 3 & $\mathrm{H}$ & A \\
\hline $\mathrm{n}$ & 3 & $\mathrm{M}$ & A \\
\hline$s$ & 1 & $\mathrm{H}$ & B \\
\hline $\mathrm{n}$ & 1 & $\mathrm{M}$ & B \\
\hline $\mathrm{n}$ & 2 & $\mathrm{H}$ & B \\
\hline s & 2 & $\mathrm{M}$ & B \\
\hline $\mathrm{n}$ & 3 & $\mathrm{H}$ & B \\
\hline $\mathrm{n}$ & 3 & $\mathrm{M}$ & B \\
\hline
\end{tabular}




\begin{tabular}{|c|c|c|c|}
\hline \multicolumn{4}{|c|}{ Pregunta: ¿Usted/tú cree(s) que checar está bien dicho en español? } \\
\hline Respuesta & Edad & Género & Nivel educativo \\
\hline$s$ & 1 & $\mathrm{H}$ & A \\
\hline$s$ & 1 & M & A \\
\hline $\mathrm{n}$ & 2 & $\mathrm{H}$ & A \\
\hline$s$ & 2 & M & A \\
\hline$s$ & 3 & $\mathrm{H}$ & A \\
\hline$s$ & 3 & $\mathrm{M}$ & A \\
\hline $\mathrm{n}$ & 1 & $\mathrm{H}$ & B \\
\hline$s$ & 1 & M & B \\
\hline $\mathrm{n}$ & 2 & $\mathrm{H}$ & B \\
\hline $\mathrm{n}$ & 2 & M & $\mathrm{B}$ \\
\hline $\mathrm{n}$ & 3 & $\mathrm{H}$ & B \\
\hline$s$ & 3 & M & $\mathrm{B}$ \\
\hline
\end{tabular}

Tabla VIII. Respuestas: $O=$ no conoce la palabra, $a=$ acoso, $b=$ bullying; $O=$ no conoce la palabra, $a=$ homosexual, $b=g a y ; 0=$ no conoce la palabra, $a=$ checar,

$$
b=\text { verificar, } c=\text { ver }
$$

\begin{tabular}{|c|c|c|c|}
\hline \multicolumn{4}{|c|}{ Pregunta: ¿Es preferible decir a) acoso o b) bullying? } \\
\hline Respuesta & Edad & Género & Nivel educativo \\
\hline $\mathrm{a}$ & 1 & $\mathrm{H}$ & A \\
\hline a & 1 & M & A \\
\hline $\mathrm{b}$ & 2 & $\mathrm{H}$ & A \\
\hline a & 2 & M & A \\
\hline$a, b$ & 3 & $\mathrm{H}$ & A \\
\hline a & 3 & M & A \\
\hline b & 1 & $\mathrm{H}$ & B \\
\hline 0 & 1 & M & B \\
\hline a & 2 & $\mathrm{H}$ & B \\
\hline 0 & 2 & M & B \\
\hline a & 3 & $\mathrm{H}$ & B \\
\hline $\mathrm{b}$ & 3 & M & B \\
\hline
\end{tabular}




\begin{tabular}{|c|c|c|c|}
\hline \multicolumn{4}{|c|}{ Pregunta: ¿̨Es preferible decir a) homosexual o b) gay? } \\
\hline Respuesta & Edad & Género & Nivel educativo \\
\hline $\mathrm{b}$ & 1 & $\mathrm{H}$ & A \\
\hline $\mathrm{b}$ & 1 & M & A \\
\hline $\mathrm{b}$ & 2 & $\mathrm{H}$ & A \\
\hline $\mathrm{b}$ & 2 & $\mathrm{M}$ & A \\
\hline $\mathrm{a}$ & 3 & $\mathrm{H}$ & A \\
\hline $\mathrm{a}$ & 3 & M & A \\
\hline$a, b$ & 1 & $\mathrm{H}$ & B \\
\hline $\mathrm{a}$ & 1 & $\mathrm{M}$ & $\mathrm{B}$ \\
\hline a & 2 & $\mathrm{H}$ & B \\
\hline $\mathrm{b}$ & 2 & M & B \\
\hline $\mathrm{a}$ & 3 & $\mathrm{H}$ & B \\
\hline $\mathrm{a}$ & 3 & M & B \\
\hline \multicolumn{4}{|c|}{ ¿En su/tu opinión es preferible decir a) checar, b) verificar o c) ver? } \\
\hline Respuesta & Edad & Género & Nivel educativo \\
\hline $\mathrm{a}$ & 1 & $\mathrm{H}$ & A \\
\hline$a, b, c$ & 1 & M & A \\
\hline b, c & 2 & $\mathrm{H}$ & A \\
\hline $\mathrm{a}$ & 2 & M & A \\
\hline $\mathrm{a}, \mathrm{bc}$ & 3 & $\mathrm{H}$ & A \\
\hline $\mathrm{b}$ & 3 & M & A \\
\hline $\mathrm{b}$ & 1 & $\mathrm{H}$ & B \\
\hline c & 1 & M & B \\
\hline $\mathrm{b}$ & 2 & $\mathrm{H}$ & B \\
\hline c & 2 & M & B \\
\hline $\mathrm{b}$ & 3 & $\mathrm{H}$ & B \\
\hline $\mathrm{a}$ & 3 & M & B \\
\hline
\end{tabular}

